\title{
Prox-regular sets and Legendre-Fenchel transform related to separation properties
}

\author{
Samir Adly*, Florent Nacrył Lionel Thibault ${ }^{\ddagger}$
}

\begin{abstract}
This paper is devoted to nonconvex/prox-regular separations of sets in Hilbert spaces. We introduce the Legendre-Fenchel $r$-conjugate of a prescribed function and $r$-quadratic support functionals and points of a given set, all associated to a positive constant $r$. By means of these concepts we obtain nonlinear functional separations for points and prox-regular sets. In addition to such functional separations, we also establish geometric separation results with balls for a prox-regular set and a strongly convex set.
\end{abstract}

Keywords. Prox-regular set, strongly convex set, Legendre-Fenchel transform, semiconvexity, quadratic support functional, geometric separation.

\section{Introduction}

The present paper is concerned with the interest of the use of the Legendre-Fenchel transform in the study of certain other crucial features for prox-regular sets which allow us to provide fundamental separation results for such sets. By means of the usual Legendre-Fenchel transform $([26,21])$, we define the concept of $r$-conjugate $f^{\star, r}$ of a function $f: X \rightarrow \mathbb{R} \cup\{-\infty,+\infty\}$ as the Legendre-Fenchel conjugate of $f+\frac{1}{2 r}\|\cdot\|^{2}$, where $X$ is a Hilbert space and $r \in] 0,+\infty]$ is a positive extended-real number. We develop the study of $r$-conjugates and we obtain diverse remarkable properties, especially for semiconvex functions. A fixed point result is also obtained for the transform $f \mapsto f^{\star, r}$. Considering the fundamental case when $f$ is the indicator function of a subset $S$ of $X$ gives rise to the quadratic functional $q_{x^{*}, r}:=\left\langle x^{*}, \cdot\right\rangle-\frac{1}{2 r}\|\cdot\|^{2}$ on $X$ for every $x^{*} \in X$. Through this, the usual notions of support linear functionals and support points in functional analysis are extended to support quadratic functionals and quadratically supported points. We study in details the properties of these new notions mainly for (uniformly) $r$-prox-regular sets in $X$. We show in particular a Bishop-Phelps ([11]) type result for the density of $r$ quadratically supported points of the set $S$ in its boundary whenever $S$ is $r$-prox-regular. With the above concepts and their properties at hands, we establish functional separation results for points outside prox-regular sets and also ball separation results (that is, separation with balls) between an $r$-prox-regular set and an $s$-strongly convex set (see Section 5 for definition).

The paper is outlined as follows. Section 2 recalls the notions and results useful for our analysis. Clarke tangent cones, normal cones and subdifferentials as well as proximal normals and proximal subdifferentials, as basic tools in our approach, are recalled in Subsection 2.1. The definition and main results needed in the paper for prox-regular sets are given in Subsection 2.2. The concepts of $r$-conjugate, quadratic support functional and quadratically supported point are defined and analyzed in Section 4. Proposition 3.1 in this section shows properties of quadratic support functionals and points, Proposition 3.4 illustrates particular features of $f^{\star, r}$ when $f$ is semiconvex, and Proposition 3.5 demonstrates the fixed point result for the transform $f \mapsto f^{\star, r}$. In Section 4 , for

\footnotetext{
*Université de Limoges, Laboratoire XLIM, 123 avenue Albert Thomas, 87060 Limoges, France. Email: samir.adly@unilim.fr

${ }^{\dagger}$ Laboratoire de Mathématiques, Physique et Systèmes, Université de Perpignan Via Domitia, 52 Avenue Paul Alduy, 66860 Perpignan. Email: florent.nacry@univ-perp.fr

¥Université de Montpellier, Institut Montpelliérain Alexander Grothendieck 34095 Montpellier CEDEX 5 France. Email: lionel.thibault@umontpellier.fr
} 
any $r$-prox-regular set in $X$ we establish in Proposition 4.1 and Theorem 4.3, functional separation type results for points at positive distances less than the radius $r$ of proxregularity. Ball separation type results between prox-regular sets and strongly convex sets are provided in Theorem 5.3 and Theorem 5.4 in the last Section 5 .

\section{Notation and preliminaries}

As usual, $\mathbb{N}$ denotes the set of integers starting from 1 and $\overline{\mathbb{R}}:=\mathbb{R} \cup\{-\infty,+\infty\}$ is the extended real-line. Throughout the paper, $X$ stands for a (real) Hilbert space not reduced to the trivial space $\{0\}$ endowed with the inner product $\langle\cdot, \cdot\rangle$ and its associated norm $\|\cdot\|:=\sqrt{\langle\cdot, \cdot\rangle}$. The open (resp. closed) ball and the sphere of $X$ centered at $x \in X$ with radius $\rho>0$ are denoted by $B(x, \rho)$ (resp. $B[x, \rho]$ ) and $S(x, \rho)$. In the particular case of the closed unit ball and the unit sphere of $X$ centered at zero, we use the following notation

$$
\mathbb{B}:=B\left[0_{X}, 1\right] \text { and } \mathbb{S}:=S\left(0_{X}, 1\right) .
$$

Now, consider any nonempty subset $S$ of $X$. The distance function $d_{S}$ from $S$ is defined as

$$
d_{S}(x)=d(x, S):=\inf _{y \in S}\|x-y\| \quad \text { for all } x \in S .
$$

The multimapping $\operatorname{Proj}_{S}: X \rightrightarrows X$ of nearest points in $S$ is defined by

$$
\operatorname{Proj}_{S}(x)=\operatorname{Proj}(S, x):=\left\{y \in S:\|x-y\|=d_{S}(x)\right\} \quad \text { for all } x \in X .
$$

Whenever the latter set is reduced to a singleton for some $\bar{x} \in X$, that is $\operatorname{Proj}_{S}(\bar{x})=\{\bar{y}\}$, the vector $\bar{y} \in S$ is denoted by $\operatorname{proj}_{S}(\bar{x})$ or $P_{S}(\bar{x})$. Let $\left.\left.r \in\right] 0,+\infty\right]$ be an extended real. Through the distance function, we can define the (open) $r$-tube of $S$ as the set $\operatorname{Tube}_{r}(S):=U_{r}(S) \backslash S$ where $U_{r}(S)$ is the (open) $r$-enlargement of $S$

$$
U_{r}(S):=\left\{x \in X: d_{S}(x)<r\right\}
$$

the (closed) $r$-exterior of $S$ as the set

$$
\operatorname{Exte}_{r}(S):=\left\{x \in X: d_{S}(x) \geq r\right\}
$$

and the set of points at exact $r$-distance to $S$

$$
D_{r}(S):=\left\{x \in X: d_{S}(x)=r\right\} .
$$

If $r=+\infty$, such sets become

$$
U_{\infty}(S):=X \quad \text { and } \operatorname{Exte}_{\infty}(S)=\emptyset=D_{\infty}(S)
$$

\subsection{Tangent and normal cones}

Throughout this paragraph, $S$ is a subset of $X$ which contains a vector $x$.

The Bouligand tangent cone $T^{B}(S ; x)$ of $S$ at $x \in S$ is defined as the set of $h \in X$ such that there exist a sequence $\left(t_{n}\right)_{n \in \mathbb{N}}$ of positive reals with $t_{n} \rightarrow 0$ and a sequence $\left(h_{n}\right)_{n \in \mathbb{N}}$ of $X$ with $h_{n} \rightarrow h$ such that

$$
x+t_{n} h_{n} \in S \text { for all } n \in \mathbb{N} .
$$

The Clarke tangent cone $T^{C}(S ; x)$ of $S$ at $x \in S$ is the set of $h \in X$ such that for every sequence $\left(x_{n}\right)_{n \in \mathbb{N}}$ of $S$ with $x_{n} \rightarrow x$, for every sequence $\left(t_{n}\right)_{n \in \mathbb{N}}$ of positive reals with $t_{n} \rightarrow 0$, there is a sequence $\left(h_{n}\right)_{n \in \mathbb{N}}$ of $X$ with $h_{n} \rightarrow h$ satisfying

$$
x_{n}+t_{n} h_{n} \in S \text { for all } n \in \mathbb{N}
$$


the negative polar cone of $T^{C}(S ; x)$ is the Clarke normal cone $N^{C}(S ; x)$ of $S$ at $x$, i.e,

$$
N^{C}(S ; x)=\left\{\zeta \in X:\langle\zeta, h\rangle \leq 0, \forall h \in T^{C}(S ; x)\right\} .
$$

Given a nonempty open set $U$ in $X$ and a function $f: U \rightarrow \overline{\mathbb{R}}$, the Clarke subdifferential $\partial_{C} f(\bar{x})$ of $f$ at a point $\bar{x} \in U$ where $f$ is finite is defined by

$$
\partial_{C} f(\bar{x}):=\left\{\zeta \in X:(\zeta,-1) \in N^{C}(\operatorname{epi} f ;(\bar{x}, f(\bar{x})))\right\},
$$

where epi $f:=\{(u, r) \in U \times \mathbb{R}: f(u) \leq r\}$ is the epigraph of $f$. By convention, $\partial_{C} f(\bar{x})=\emptyset$ when $f(\bar{x})$ is not finite. Defining the indicator function $\psi_{S}$ of the set $S$ by

$$
\psi_{S}\left(x^{\prime}\right):= \begin{cases}0 & \text { if } x^{\prime} \in S \\ +\infty & \text { if } x^{\prime} \in X \backslash S\end{cases}
$$

one has $\partial_{C} \psi_{S}\left(x^{\prime}\right)=N^{C}\left(S ; x^{\prime}\right)$ for all $x^{\prime} \in S$.

Besides the above notions of Clarke normal cone and subdifferential, let us introduce the proximal normal ones. The proximal normal cone of $S$ at $x \in S$, denoted by $N^{P}(S ; x)$, is defined as (see, e.g., [33])

$$
N^{P}(S ; x):=\left\{v \in X: \exists r>0, x \in \operatorname{Proj}_{S}(x+r v)\right\} .
$$

By convention, one puts

$$
T^{C}\left(S ; x^{\prime}\right)=\emptyset \quad \text { and } \quad N^{C}\left(S ; x^{\prime}\right)=N^{P}\left(S ; x^{\prime}\right)=\emptyset \quad \text { for all } x^{\prime} \in X \backslash S .
$$

It is not difficult to see that $v \in N^{P}(S ; x)$ if and only if there is a real $r>0$ such that

$$
\left\langle v, x^{\prime}-x\right\rangle \leq \frac{1}{2 r}\left\|x^{\prime}-x\right\|^{2} \quad \text { for all } x^{\prime} \in S ;
$$

in such a case we will say that $v$ is a proximal normal to $S$ at $x$ with constant $r>0$. It is known and not difficult to see that

$$
N^{P}(S ; x) \subset N^{C}(S ; x) .
$$

According to the definition of the proximal normal cone, we notice that, for any $v \in X$ such that $\operatorname{Proj}_{S}(v) \neq \emptyset$, we have

$$
v-w \in N^{P}(S ; w) \text { for all } w \in \operatorname{Proj}_{S}(v) .
$$

Coming back to the above function $f: U \rightarrow \overline{\mathbb{R}}$ defined on the nonempty open set $U$ of $X$, a vector $\zeta \in X$ is said to be a proximal subgradient of $f$ at a point $\bar{x} \in U$ with $f(\bar{x})$ finite, provided there are a real $\sigma \geq 0$ and a real $\eta>0$ with $B(\bar{x}, \eta) \subset U$ such that

$$
\langle\zeta, y-\bar{x}\rangle \leq f(y)-f(\bar{x})+\sigma\|y-\bar{x}\|^{2} \quad \text { for all } y \in B(\bar{x}, \eta),
$$

which is known to be equivalent to $(\zeta,-1) \in N^{P}($ epi $f ;(\bar{x}, f(\bar{x})))$. The set $\partial_{P} f(\bar{x})$ of all proximal subgradients of $f$ at $\bar{x}$ is the proximal subdifferential of $f$ at $\bar{x}$. Like for the Clarke subdifferential, one sets $\partial_{P} f(\bar{x})=\emptyset$ whenever $f$ is not finite at $\bar{x}$.

If $S$ is convex, it is known (and easily seen) that the two normal cones $N^{P}(S ; x)$ and $N^{C}(S ; x)$ coincide with the normal cone in the sense of convex analysis, that is,

$$
N^{P}(S ; x)=N^{C}(S ; x)=\{\zeta \in X:\langle\zeta, y-x\rangle \leq 0, \forall y \in S\} .
$$

If $f: U \rightarrow X$ is a convex function defined on a nonempty open convex subset $U$ of $X$, then the two above subdifferentials coincide on $U$ with the subdifferential in the sense of convex analysis, i.e., for every $\bar{x} \in U$,

$$
\partial_{P} f(\bar{x})=\partial_{C} f(\bar{x})=\{\zeta \in X:\langle\zeta, y-\bar{x}\rangle \leq f(y)-f(\bar{x}), \forall y \in U\}=: \partial f(\bar{x}) .
$$




\subsection{Prox-regular sets in the Hilbert setting}

This succinct paragraph is devoted to the needed basic features on prox-regularity which is at the heart of the present paper. Prox-regular sets (a term coined in [31]) has a long history which goes back to the famous work [20] by H. Federer with the class of positively reached sets in $\mathbb{R}^{n}$. Over the years, various names have been introduced in different contexts to refer to such sets: weakly convex ([37]) or proximally smooth sets $([16])$ are commonly used in Hilbert setting; for other names we refer to the work [17]. Studies of prox-regular sets in Banach spaces can be found in $[9,10,6]$. Besides those theoretical developments, let us mention that prox-regular sets have also been sucessfully involved in many concrete problems as the celebrated Moreau's sweeping process (see, e.g., the survey [29] and the references therein). For more details, in both theoretical and concrete approaches, one can refer for instance to [31], the survey [17] and the book [34] along with the references therein. We also refer to $[1,2,35]$ for various stability properties.

Definition 2.1 Let $S$ be a nonempty closed subset of $X$ and $r \in] 0,+\infty]$. One says that $S$ is $r$-prox-regular (or uniformly prox-regular with constant $r$ ) whenever, for every $x \in S$, for every $v \in N^{P}(S ; x) \cap \mathbb{B}$ and for every real $\left.\left.t \in\right] 0, r\right]$, one has

$$
x \in \operatorname{Proj}_{S}(x+t v) .
$$

Given a closed subset $S \subset X, x \in S$ and $v \in N^{P}(S ; x)$ with $\|v\|=1$, it is known and easily seen that for every real $t>0$ one has

$$
x \in \operatorname{Proj}_{S}(x+t v) \Leftrightarrow S \cap B(x+t v, t)=\emptyset .
$$

In such a case, one says that the unit normal proximal vector $v$ to $S$ at $x$ is realized by the $t$-ball $B(x+t v, t)$.

The following theorem provides some useful characterizations and properties of uniform prox-regular sets for which we refer to [31, 17, 34].

Theorem 2.1 Let $S$ be a nonempty closed subset of $X$ and let $r \in] 0,+\infty]$. Consider the following assertions.

(a) The set $S$ is r-prox-regular.

(b) For all $x, x^{\prime} \in S$, for all $v \in N^{P}(S ; x)$, one has

$$
\left\langle v, x^{\prime}-x\right\rangle \leq \frac{1}{2 r}\|v\|\left\|x-x^{\prime}\right\|^{2} .
$$

(c) On the open set $U_{r}(S)$ the mapping $\operatorname{proj}_{S}$ is well-defined, and for every real $\left.s \in\right] 0, r[$, for all $x, x^{\prime} \in U_{s}(S)$,

$$
\left\|\operatorname{proj}_{S}(x)-\operatorname{proj}_{S}\left(x^{\prime}\right)\right\| \leq \frac{1}{1-(s / r)}\left\|x-x^{\prime}\right\| .
$$

(d) For any $u \in \operatorname{Tube}_{r}(S)$ such that $\operatorname{proj}_{S}(u)$ exists, one has

$$
\operatorname{proj}_{S}(u)=\operatorname{proj}_{S}\left(\operatorname{proj}_{S}(u)+t \frac{u-\operatorname{proj}_{S}(u)}{\left\|u-\operatorname{proj}_{S}(u)\right\|}\right) \quad \text { for allt } \in[0, r[.
$$

(e) The function $d_{S}^{2}$ is $C^{1,1}$ on $U_{r}(S)$ and

$$
\nabla d_{S}^{2}(x)=2\left(x-\operatorname{proj}_{S}(x)\right) \quad \text { for all } x \in U_{r}(S) .
$$

(f) The set $S$ is tangentially regular as well as normally regular in the sense that

$$
T^{B}(S ; x)=T^{C}(S ; x) \quad \text { and } \quad N^{P}(S ; x)=N^{C}(S ; x) \quad \text { for all } x \in X .
$$

Further, one has

$$
\partial_{P} d_{S}(x)=\partial_{C} d_{S}(x) \quad \text { for all } x \in U_{r}(S) .
$$

Then, the assertions $(a),(b),(c),(d),(e)$ are pairwise equivalent and each one implies the assertion $(f)$. 
When $S$ is $r$-prox-regular, according to the above assertion (f) we will just write $N(S ; \cdot)$ in place of any of $N^{P}(S ; \cdot)$ and $N^{C}(S ; \cdot)$, except when we need to precise the type of normal cone we are working with. If $C$ is $\infty$-prox-regular in $X$ (i.e., nonempty closed and convex) then $N(C ; \cdot)$ coincides with the normal cone in the sense of convex analysis, that is,

$$
N(C ; x)=\{\zeta \in X:\langle\zeta, y-x\rangle \leq 0, \forall y \in C\} \quad \text { for all } x \in C
$$

Remark 2.1 In addition to $(d)$ in the above theorem, it is worth noticing that for an $r$-prox-regular subset of $X$ with $r \in] 0,+\infty[$, we have the crucial inclusion

$$
\operatorname{proj}_{S}(u) \in \operatorname{Proj}_{S}\left(\operatorname{proj}_{S}(u)+r \frac{u-\operatorname{proj}_{S}(u)}{\left\|u-\operatorname{proj}_{S}(u)\right\|}\right) \quad \text { for all } u \in \operatorname{Tube}_{r}(S) .
$$

\section{Quadratic support functional and Legendre-Fenchel $r$-conjugate}

Consider for a moment a nonempty closed convex set $C$ of $X$ and $\bar{x} \in \operatorname{bdry} C$. When the non-nullity property

$$
N(C ; \bar{x}) \neq\{0\}
$$

holds, we can see that it translates a geometrical separation between the set $C$ and the vector $\bar{x}$. Indeed, with the definition of the normal cone in the sense of convex analysis at hands (see (2.3)), we observe that for any $x^{\star} \in N(C ; \bar{x}) \backslash\{0\}$,

$$
\left\langle x^{\star}, c\right\rangle \leq\left\langle x^{\star}, \bar{x}\right\rangle \quad \text { for all } c \in C,
$$

and this can be rewritten with the help of the support function $\sigma(\cdot, C):=\sup _{c \in C}\langle\cdot, c\rangle$ as

$$
C \subset\left\{\left\langle x^{\star}, \cdot\right\rangle \leq \sigma\left(x^{\star}, C\right)\right\} \quad \text { and } \quad\left\langle x^{\star}, \bar{x}\right\rangle \geq \sigma\left(x^{\star}, C\right)
$$

It should be noted that the latter inequality is in fact an equality, that is

$$
\left\langle x^{\star}, \bar{x}\right\rangle=\sigma\left(x^{\star}, C\right) .
$$

Coming back to an $r$-prox-regular set $S$ of $X$ for some real $r>0$, let us take a look at the meaning of the non-nullity similar property

$$
N^{P}(S ; \bar{x}) \neq\{0\} \quad \text { for some } \bar{x} \in \operatorname{bdry} S .
$$

Fix any $x^{\star} \in N^{P}(S ; \bar{x}) \backslash\{0\}$ with $\left\|x^{\star}\right\|=1$. According to the $r$-prox-regularity property (see Theorem 2.1), we have

$$
\left\langle x^{\star}, u-\bar{x}\right\rangle \leq \frac{1}{2 r}\|u-\bar{x}\|^{2} \quad \text { for all } u \in S,
$$

or equivalently,

$$
\left\langle x^{\star}+\frac{\bar{x}}{r}, u\right\rangle-\frac{\|u\|^{2}}{2 r} \leq\left\langle x^{\star}+\frac{\bar{x}}{r}, \bar{x}\right\rangle-\frac{\|\bar{x}\|^{2}}{2 r} \quad \text { for all } u \in S .
$$

Setting $y^{\star}:=x^{\star}+\frac{\bar{x}}{r} \neq \frac{\bar{x}}{r}$ and $\varphi_{S, r}\left(y^{\star}\right):=\sup _{u \in S}\left(\left\langle y^{\star}, u\right\rangle-\frac{\|u\|^{2}}{2 r}\right)$, we arrive to

$$
S \subset\left\{\left\langle y^{\star}, \cdot\right\rangle-\frac{\|\cdot\|^{2}}{2 r} \leq \varphi_{S, r}\left(y^{\star}\right)\right\} \quad \text { and } \quad\left\langle y^{\star}, \bar{x}\right\rangle-\frac{\|\bar{x}\|^{2}}{2 r} \geq \varphi_{S, r}\left(y^{\star}\right) .
$$


Defining the quadratic functional $q_{y^{*}, r}: X \rightarrow \mathbb{R}$ by

$$
q_{y^{\star}, r}(x):=\left\langle y^{\star}, x\right\rangle-\frac{\|x\|^{2}}{2 r} \text { for all } x \in X,
$$

we observe in the same line as (3.1) that

$$
q_{y^{\star}, r}(\bar{x})=\varphi_{S, r}\left(y^{\star}\right) .
$$

This leads to introduce the following definition.

Definition 3.1 Let $S$ be a nonempty closed subset of $X$ and $r \in] 0,+\infty]$ be an extended real. Given $u^{\star} \in X$, we say that $q_{u^{\star}, r}$ is an $r$-quadratic support functional of $S$ whenever there exists $\bar{x} \in S$ such that

$$
q_{u^{\star}, r}(\bar{x}):=\left\langle u^{\star}, \bar{x}\right\rangle-\frac{1}{2 r}\|\bar{x}\|^{2}=\sup _{x \in S}\left(\left\langle u^{\star}, x\right\rangle-\frac{1}{2 r}\|x\|^{2}\right)=\sup _{x \in S} q_{u^{\star}, r}(x)=: \varphi_{S, r}\left(u^{\star}\right) .
$$

In such a case, $u^{\star}$ is said to quadratically support $S$ at $\bar{x}$. We say that $\bar{x} \in S$ is an $r$-quadratic support point of $S$ whenever there exists $u^{\star} \in X$ with $u^{\star} \neq \frac{\bar{x}}{r}$ such that $q_{u^{\star}, r}$ supports $S$ at $\bar{x}$. The set of all $r$-quadratic support points of $S$ is denoted by $\operatorname{supp}_{r}(S)$.

Whenever $r=+\infty$, the above definition is reduced to saying that $\left\langle u^{*}, \cdot\right\rangle$ (resp. $\bar{x}$ ) is a support linear functional (resp. support point) of $S$ in the usual sense of functional analysis. Recall that $\left\langle u^{\star}, \cdot\right\rangle$ with $u^{\star} \in X$ is a support linear functional to a nonempty closed subset $S$ of $X$ provided there is some $\bar{x} \in S$ such that

$$
\left\langle u^{\star}, \bar{x}\right\rangle=\sup _{x \in S}\left\langle u^{\star}, x\right\rangle=: \sigma\left(u^{\star}, S\right),
$$

and if $u^{\star} \neq 0$, such a point $\bar{x} \in S$ is said to be a support point of $S$. If in addition $S$ is convex, it is readily seen that the latter equality is equivalent to the following inclusion

$$
u^{\star} \in N(S ; \bar{x}) \text {. }
$$

Through the next proposition, we see that the notions of $r$-quadratic support functionals and points are related to the proximal normal cone of $S$.

Proposition 3.1 Let $S$ be a closed subset of $\left.\left.X, u^{\star} \in X, \bar{x} \in S, r \in\right] 0,+\infty\right]$. The following hold.

(a) The functional $q_{u^{\star}, r}$ is an $r$-quadratic support functional of $S$ if and only if there exists $z \in S$ such that

$$
\left\langle u^{\star}-\frac{z}{r}, x-z\right\rangle \leq \frac{1}{2 r}\|x-z\|^{2} \quad \text { for all } x \in S .
$$

$\left(a^{\prime}\right)$ The vector $\bar{x}$ is an r-quadratic support point of $S$ supported by $q_{u^{\star}, r}$ if and only if $u^{\star} \neq \frac{\bar{x}}{r}$ and

$$
\left\langle u^{\star}-\frac{\bar{x}}{r}, x-\bar{x}\right\rangle \leq \frac{1}{2 r}\|x-\bar{x}\|^{2} \quad \text { for all } x \in S .
$$

(b) If $q_{u^{\star}, r}$ is an $r$-quadratic support functional of $S$, then there exists $z \in S$ such that $u^{\star}-\frac{z}{r}$ is a proximal normal to $S$ at $z$ with constant $r$.

$\left(b^{\prime}\right)$ If $\bar{x}$ is an $r$-quadratic support point of $S$ supported by the quadratic functional $q_{u^{\star}, r}$, then there exists a nonzero proximal normal $v^{\star}$ to $S$ at $\bar{x}$ with constant $r$ such that

$$
u^{\star}=v^{\star}+\frac{\bar{x}}{r} .
$$

(c) If $q_{u^{\star}, r}$ is an $r$-quadratic support functional of $S$, then for every real $\alpha>0, q_{\alpha u^{\star}, r / \alpha}$ is an $\frac{r}{\alpha}$-quadratic support functional of $S$.

$\left(c^{\prime}\right)$ If $\bar{x}$ is an r-quadratic support point of $S$ supported by the quadratic functional $q_{u^{\star}, r}$, 
then $\bar{x}$ is an $\frac{r}{\alpha}$-quadratic support point of $S$ supported by the quadratic functional $q_{\alpha u^{\star}, r / \alpha}$.

If in addition the set $S$ is r-prox-regular, one has:

(d) $q_{u^{\star}, r}$ is an $r$-quadratic support functional of $S$ whenever there exists $z \in S$ such that

$$
u^{\star} \in N(S ; z) \cap \mathbb{B}+\frac{z}{r} .
$$

$\left(d^{\prime}\right)$ The vector $\bar{x}$ is an $r$-quadratic support point of $S$ supported by the $r$-quadratic functional $q_{u^{\star}, r}$ whenever $u^{\star} \neq \frac{\bar{x}}{r}$ and

$$
u^{\star} \in N(S ; \bar{x}) \cap \mathbb{B}+\frac{\bar{x}}{r} .
$$

(e) One has the equality

$$
\operatorname{supp}_{r}(S)=\left\{x \in \operatorname{bdry}_{X} S: N(S ; x) \neq\{0\}\right\} .
$$

Proof. $(a)$ and $\left(a^{\prime}\right)$ : To establish $(a)$, it suffices to fix any $z \in S$ and to note that for every $x \in S$,

$$
\begin{aligned}
\left\langle u^{\star}, x\right\rangle-\frac{1}{2 r}\|x\|^{2} \leq\left\langle u^{\star}, z\right\rangle-\frac{1}{2 r}\|z\|^{2} & \Leftrightarrow\left\langle u^{\star}, x-z\right\rangle \leq \frac{1}{2 r}\left(\|x\|^{2}-\|z\|^{2}\right) \\
& \Leftrightarrow\left\langle u^{\star}, x-z\right\rangle \leq \frac{1}{2 r}\left(\|x-z\|^{2}+2\langle x-z, z\rangle\right) \\
& \Leftrightarrow\left\langle u^{\star}-\frac{z}{r}, x-z\right\rangle \leq \frac{1}{2 r}\|x-z\|^{2} .
\end{aligned}
$$

The equivalence provided by $\left(a^{\prime}\right)$ is a direct consequence of what precedes.

$(b)$ and $\left(b^{\prime}\right)$ : The implication claimed by $(b)$ is a direct consequence of $(a)$ and of the characterization (2.1) of proximal normal vectors. Similarly, $\left(b^{\prime}\right)$ follows from both $\left(a^{\prime}\right)$ and (2.1).

$(c)$ and $\left(c^{\prime}\right)$ : These assertions follows from $(a)$ and $\left(a^{\prime}\right)$ respectively.

Now, assume that the set $S$ is $r$-prox-regular.

$(d)$ and $\left(d^{\prime}\right)$ : It is enough to establish $(d)$. If there is $z \in S$ such that

$$
u^{\star} \in N(S ; z) \cap \mathbb{B}+\frac{z}{r},
$$

then Theorem 2.1 ensures that

$$
\left\langle u^{\star}-\frac{z}{r}, x-z\right\rangle \leq \frac{1}{2 r}\|x-z\|^{2} \quad \text { for all } x \in S .
$$

Applying (a) yields that $q_{u^{\star}, r}$ is an $r$-quadratic support functional of $S$.

(e) The inclusion

$$
\operatorname{supp}_{r}(S) \subset\left\{x \in \operatorname{bdry}_{X} S: N(S ; x) \neq\{0\}\right\}
$$

is a direct consequence of $\left(b^{\prime}\right)$. In order to establish the converse inclusion, fix any $\bar{x} \in$ $\operatorname{bdry}_{X} S$ such that $N(S ; \bar{x}) \neq\{0\}$. Let any $v^{\star} \in N(S ; \bar{x})$ with $\left\|v^{\star}\right\|=1$. We have

$$
u^{\star}:=v^{\star}+\frac{\bar{x}}{r} \in N(S ; \bar{x}) \cap \mathbb{B}+\frac{\bar{x}}{r}
$$

and $u^{\star} \neq \frac{\bar{x}}{r}$ (otherwise $v^{\star}=0$ ). It follows from $\left(d^{\prime}\right)$ that $\bar{x}$ is an $r$-quadratic support point of the set $S$.

Remark 3.1 Consider any nonempty closed set $S$ of $X$ with $S \neq X$. According to [27, Theorem 2.10 and Proposition 2.6], we know that the set

$$
\left\{x \in \operatorname{bdry} S: N^{F}(S ; x) \neq\{0\}\right\}
$$

is dense in bdry $S$, where $N^{F}(S ; \cdot)$ denotes the Fréchet normal cone of $S$ (see, e.g., [27]). Taking into account the property $(e)$ in the above proposition and assuming in addition that $S$ is $r$-prox-regular for some $r \in] 0,+\infty]$, we arrive to the density of $\operatorname{supp}_{r}(S)$ in the boundary of $S$. This can be seen as an prox-regular extension of the celebrated BishopPhelps theorem (see [11]). 
Let $S$ be an $r$-prox-regular subset of $X$ and $\bar{x} \in \operatorname{Tube}_{r}(S)$. Thanks to the inclusion

$$
\mathbb{R}_{+}\left(\bar{x}-\operatorname{proj}_{S}(\bar{x})\right) \subset N\left(S ; \operatorname{proj}_{S}(\bar{x})\right)
$$

we have

$$
\frac{\bar{x}-\operatorname{proj}_{S}(\bar{x})}{d_{S}(\bar{x})}+\frac{\operatorname{proj}_{S}(\bar{x})}{r} \in N\left(S ; \operatorname{proj}_{S}(\bar{x})\right) \cap \mathbb{B}+\frac{\operatorname{proj}_{S}(\bar{x})}{r} .
$$

Denoting

$$
\Gamma_{S, r}(\bar{x}):=\left(\frac{1}{r}-\frac{1}{d_{S}(\bar{x})}\right) \operatorname{proj}_{S}(\bar{x})+\frac{\bar{x}}{d_{S}(\bar{x})},
$$

we see that $q_{\Gamma_{S, r}(\bar{x}), r}$-quadratically supports $S$ at $\operatorname{proj}_{S}(\bar{x})$ according to $(d)$ in Proposition 3.1 above. Since $\bar{x} \in \operatorname{Tube}_{r}(S)$, we must have $\Gamma_{S, r}(\bar{x}) \neq \frac{\operatorname{proj}_{S}(\bar{x})}{r}$ (otherwise, $\bar{x}=\operatorname{proj}_{S}(\bar{x})$ which is a contradiction), and then $\left(d^{\prime}\right)$ above says that $\operatorname{proj}_{S}^{r}(\bar{x})$ is an $r$-quadratic support point of $S$ quadratically supported by $q_{\Gamma_{S, r}(\bar{x}), r}$.

We summarize those features in the following proposition.

Proposition 3.2 Let $S$ be an $r$-prox-regular set of $X$ with $r \in] 0,+\infty]$ and $x \in \operatorname{Tube}_{r}(S)$. The following hold:

(a) $q_{\Gamma_{S, r}(x), r}$-quadratically supports $S$ at $\operatorname{proj}_{S}(x)$;

(b) $\operatorname{proj}_{S}(x)$ is an r-quadratic support point of $S$ supported by $q_{\Gamma_{S, r}(x), r}$.

The quantity in Definition 3.1

$$
\varphi_{S, r}\left(x^{\star}\right):=\sup _{x \in S}\left(\left\langle x^{\star}, x\right\rangle-\frac{\|x\|^{2}}{2 r}\right)
$$

can be seen as a particular Legendre-Fenchel conjugate since

$$
\varphi_{S, r}=\left(\psi_{S}+\frac{\|\cdot\|^{2}}{2 r}\right)^{\star}
$$

Let us point out here that $\varphi_{S, 1}$ (which is sometimes called Asplund function) is greatly involved in convex analysis and approximation theory (see, e.g., [4, 8, 24, 25]). Various and numerous properties of $\varphi_{S, r}$ are given in our paper [3]. In particular, it is shown that such a function is the right tool to extend fundamental metric properties known in Convex Analysis to the framework of Variational Analysis of prox-regular sets. The important role of $\varphi_{S, r}$ leads us to introduce the following definition for any function.

Definition 3.2 Given a function $f: X \rightarrow \overline{\mathbb{R}}$ and an extended real $r \in] 0,+\infty]$, one defines the Legendre-Fenchel $r$-conjugate $f^{\star, r}: X \rightarrow \mathbb{R} \cup\{-\infty,+\infty\}$ by

$$
f^{\star, r}\left(x^{\star}\right):=\left(f+\frac{1}{2 r}\|\cdot\|^{2}\right)^{\star}\left(x^{\star}\right)=\sup _{x \in X}\left(\left\langle x^{\star}, x\right\rangle-\frac{1}{2 r}\|x\|^{2}-f(x)\right) \quad \text { for all } x^{\star} \in X .
$$

The following proposition states a list of properties which can be verified in a straightforward way.

Proposition 3.3 Let $f: X \rightarrow \overline{\mathbb{R}}$ be a function and $r>0$ be an extended real. The following hold.

(a) One has the equality $f^{\star, r}(0)=-\inf _{X}\left(f+\frac{1}{2 r}\|\cdot\|^{2}\right)$.

(b) One has the equivalences

$$
-\infty \in f^{\star, r}(X) \Leftrightarrow f \equiv+\infty \Leftrightarrow f^{\star, r} \equiv-\infty .
$$

(c) If the function $f^{\star, r}$ is proper, then $f$ is proper.

(d) For every $\left(x^{\star}, t\right) \in X \times \mathbb{R}$, one has the equivalence

$$
\left(x^{\star}, t\right) \in \operatorname{epi} f^{\star, r} \Leftrightarrow\left\langle x^{\star}, \cdot\right\rangle-\frac{1}{2 r}\|\cdot\|^{2}-t \leq f(\cdot) .
$$


(e) The function $f$ has no continuous $r$-quadratic minorants if and only if $f^{\star, r} \equiv+\infty$.

$(f)$ If $\operatorname{dom} f^{\star, r} \neq \emptyset$, then $f$ is bounded from below on each bounded subset of $X$.

(g) The function $f^{\star, r}$ is convex and lower semicontinuous.

(h) For all $x^{\star} \in X$, one has

$$
\begin{aligned}
f^{\star, r}\left(x^{\star}\right) & =\sup _{x \in \operatorname{dom} f}\left(\left\langle x^{\star}, x\right\rangle-\frac{1}{2 r}\|x\|^{2}-f(x)\right) \\
& =\sup _{(x, t) \in \operatorname{epi} f}\left(\left\langle x^{\star}, x\right\rangle-\frac{1}{2 r}\|x\|^{2}-t\right) .
\end{aligned}
$$

(i) If $f$ is a proper function, then one has the following Fenchel-Young inequality

$$
f(x)+f^{\star, r}\left(x^{\star}\right) \geq\left\langle x^{\star}, x\right\rangle-\frac{1}{2 r}\|x\|^{2} \quad \text { for all } x^{\star}, x \in X .
$$

The following example will be crucial in order to get fixed points of for the $r$-conjugate transforms $f \mapsto f^{\star, r}$ with $r>0$ (see Proposition 3.5).

Example 1 Let $S$ be a nonempty subset of $X$ and let $r>0$ be a positive real. Fix any real $\alpha>-\frac{1}{2 r}$ and set $f:=\psi_{S}+\alpha\|\cdot\|^{2}$. From the very definition of $r$-conjugate, we see with $\rho:=\frac{r}{2 \alpha r+1}>0$

$$
f^{\star, r}\left(x^{\star}\right)=\varphi_{S, \rho}\left(x^{\star}\right)=\frac{\rho}{2}\left\|x^{\star}\right\|^{2}-\frac{1}{2 \rho} d_{S}^{2}\left(\rho x^{\star}\right) \quad \text { for all } x^{\star} \in X .
$$

In particular, with $S=X$ we obtain

$$
\left(\alpha\|\cdot\|^{2}\right)^{\star, r}=\frac{r}{2(2 r \alpha+1)}\|\cdot\|^{2} .
$$

As usual, a function $f: X \rightarrow \overline{\mathbb{R}}$ is said to be proper if its effective domain $\operatorname{dom} f:=$ $\{x \in X: f(x)<+\infty\}$ is nonempty (i.e., $f \not \equiv+\infty$ ) along with $f(X) \subset \mathbb{R} \cup\{+\infty\}$. Recall that if $f$ is proper then the celebrated Fenchel-Moreau theorem (see, e.g., [12, 28, 34]) asserts that $f$ is lower semicontinuous and convex if and only if $f=f^{* *}$.

In the statement of the next proposition, the concepts of infimal convolution and semiconvexity will be involved. Taking two functions $f, g: X \rightarrow \mathbb{R} \cup\{+\infty\}$, we recall (see [28]) that the infimal convolution $f \square g: X \rightarrow \overline{\mathbb{R}}$ of $f$ and $g$ is defined by

$$
(f \square g)(x):=\inf _{y \in X}(f(x-y)+g(y)) \quad \text { for all } x \in X .
$$

The following definition concerns the second concept of semiconvexity (see,e.g., [14]).

Definition 3.3 A function $f: C \rightarrow \mathbb{R} \cup\{+\infty\}$ defined on a nonempty convex subset $C$ of $X$ is said to be $\sigma$-semiconvex (on $C$ ) for some $\sigma \in \mathbb{R}_{+}:=[0,+\infty[$ if

$$
f(t x+(1-t) y) \leq t f(x)+(1-t) f(y)+\frac{\sigma}{2} t(1-t)\|x-y\|^{2},
$$

for all $x, y \in C$ and for all $t \in] 0,1\left[\right.$, or equivalently if $f+\frac{\sigma}{2}\|\cdot\|^{2}$ is convex on $C$. The function $f$ is said to be semiconvex near a point if it is semiconvex on a ball centered at that point.

Recall that if $f$ is $\sigma$-semiconvex near a point $x$, then for some real $\eta>0$

$$
\partial_{P} f(x)=\partial_{C} f(x)=\left\{\zeta \in \mathcal{H}:\langle\zeta, h\rangle \leq f(x+h)-f(x)+\frac{\sigma}{2}\|h\|^{2} \forall h \in B(x, \eta)\right\} .
$$

Related to the semiconvexity property we also have. 
Proposition 3.4 Let $f: X \rightarrow \overline{\mathbb{R}}$ be a function and $r>0$ be an extended real.

(j) If $f$ is $r^{-1}$-semiconvex and lower semicontinuous on $X$ with $f(X) \subset \mathbb{R} \cup\{+\infty\}$, then one has

$$
f(x)=\sup _{x^{\star} \in X}\left(q_{x^{\star}, r}(x)-f^{\star, r}\left(x^{\star}\right)\right) \quad \text { for all } x \in X .
$$

(k) If $f$ is proper, then $f$ is $r^{-1}$-semiconvex and lower semicontinuous on $X$ if and only if $f=\left(f^{\star, r}\right)^{\star}-\frac{1}{2 r}\|\cdot\|^{2}$.

(l) If $f$ is proper, $r^{-1}$-semiconvex and lower semicontinuous on $X$, then one has

$$
\left(x, u-\frac{1}{r} x\right) \in \operatorname{gph} \partial_{C} f \Leftrightarrow(u, x) \in \operatorname{gph} \partial f^{\star, r} .
$$

(m) $\left(f^{\star, r}\right)^{\star}=\left(f+\frac{1}{2 r}\|\cdot\|^{2}\right)^{\star \star}$.

(n) $\left(f^{\star, r}\right)^{\star}=f+\frac{1}{2 r}\|\cdot\|^{2}$ if and only if $f$ is proper, $r^{-1}$-semiconvex and lower semicontinuous.

(o) $\left(f^{\star}\right)^{\star, r}=\left(f \square \frac{r}{2}\|\cdot\|^{2}\right)^{\star \star}$.

(p) $\left(f^{\star, r}\right)^{\star}=\left(f \square \frac{r}{2}\|\cdot\|^{2}\right)$ if and only if $\left(f \square \frac{r}{2}\|\cdot\|^{2}\right)$ is proper, convex and lower semicontinuous.

Proof. $(j)$ Under the assumption in $(j)$ the function $g(\cdot):=f(\cdot)+\frac{1}{2 r}\|\cdot\|^{2}$ is proper, lower semicontinuous and convex. Fix any $x \in X$. According to the Fenchel-Moreau theorem, we may write

$$
g(x)=\sup _{x^{\star} \in X}\left(\left\langle x^{\star}, x\right\rangle-g^{\star}\left(x^{\star}\right)\right),
$$

or equivalently

$$
f(x)+\frac{1}{2 r}\|x\|^{2}=\sup _{x^{\star} \in X}\left(\left\langle x^{\star}, x\right\rangle-\left(f+\frac{1}{2 r}\|\cdot\|^{2}\right)^{\star}\left(x^{\star}\right)\right) .
$$

Consequently, we derive

$$
f(x)=\sup _{x^{\star} \in X}\left(\left\langle x^{\star}, x\right\rangle-\frac{1}{2 r}\|x\|^{2}-f^{\star, r}\left(x^{\star}\right)\right)
$$

which is the desired equality.

(k) It suffices to apply again the Fenchel-Moreau theorem.

(l) It suffices to use the fact that for a proper convex lower semicontinuous function $h: X \rightarrow \overline{\mathbb{R}}$, we have (see, e.g., [28, Proposition 10.1]) for every $x, u \in X$

$$
(x, u) \in \operatorname{gph} \partial h \Leftrightarrow(u, x) \in \operatorname{gph} \partial h^{\star} .
$$

It remains to establish assertions $(m)-(p)$. Note that $(n)$ (resp. $(p))$ is a direct consequence of the Fenchel-Moreau theorem and the equality provided by $(m)$ (resp. $(o)$ ). According to (3.3), we can write

$$
\left(f^{\star, r}\right)^{\star}=\left[\left(f+\frac{1}{2 r}\|\cdot\|^{2}\right)^{\star}\right]^{\star}=\left(f+\frac{1}{2 r}\|\cdot\|^{2}\right)^{\star \star},
$$

which is $(m)$. Invoking again (3.3), we have

$$
\left(f^{\star}\right)^{\star, r}=\left(f^{\star}+\frac{1}{2 r}\|\cdot\|^{2}\right)^{\star}=\left(f^{\star}+g^{\star}\right)^{\star},
$$

where $g(\cdot):=\frac{r}{2}\|\cdot\|^{2}$. It remains to apply the equality $f^{\star}+g^{\star}=(f \square g)^{\star}$ (see, e.g., $[28$, $(6.15)$, p. 45], [34]) to get the desired equality in $(o)$. This finishes the proof of the proposition.

We establish now the fixed point result for the $r$-conjugate transform.

Proposition 3.5 Given a function $f: X \rightarrow \overline{\mathbb{R}}$ and a real $r>0$, one has

$$
f^{\star, r}(\cdot)=f(\cdot) \Leftrightarrow f(\cdot)=\frac{-1+\sqrt{1+4 r^{2}}}{4 r}\|\cdot\|^{2} .
$$


Proof. Fix any real $r>0$. Let us consider the function $g: \mathbb{R} \rightarrow \mathbb{R}$ defined by

$$
g(t):=4 r t^{2}+2 t-r \quad \text { for all } t \in \mathbb{R} .
$$

It is a routine to observe that $g$ has exactly two zeros, namely

$$
\alpha:=\frac{-1+\sqrt{1+4 r^{2}}}{4 r} \geq 0 \text { and } \beta:=\frac{-1-\sqrt{1+4 r^{2}}}{4 r} .
$$

$\Leftarrow$, Assume that $f(\cdot)=\alpha\|\cdot\|^{2}$. Observe first that the equality $g(\alpha)=0$ entails $\frac{\rho}{2}=\alpha$ with $\rho:=\frac{r}{2 \alpha r+1}$. Then, using the formulae (3.4) provided by Example 1 furnishes

$$
f^{\star, r}(\cdot)=\frac{\rho}{2}\|\cdot\|^{2}=\alpha\|\cdot\|^{2}=f(\cdot) .
$$

$\Rightarrow$, Assume that $f^{\star, r}(\cdot)=f(\cdot)$. According to Proposition 3.3(b), $-\infty \notin f^{\star, r}(X)=f(X)$ (otherwise $f \neq f^{\star, r}$ ) and then $f \not \equiv+\infty$. Thus, $f$ is a proper function and this allows us to apply the extended Fenchel-Young inequality in Proposition 3.4(i) above to obtain

$$
f(x) \geq \frac{2 r-1}{4 r}\|x\|^{2} \quad \text { for all } x \in X .
$$

Now, let us consider the function $h: I:=]-\frac{1}{2 r},+\infty[\rightarrow \mathbb{R}$ given by

$$
h(t):=\frac{r}{2} \frac{1}{2 r t+1} \quad \text { for all } t \in I
$$

Note that $\alpha$ is the only one fixed point for $h$. Further, it is clear that $h$ is decreasing on $I$ along with $h(I) \subset I$. Hence, with $u_{0}:=\frac{2 r-1}{4 r} \in I$, we can set

$$
u_{n}:=h\left(u_{n-1}\right) \quad \text { for all } n \in \mathbb{N} .
$$

Rewriting (3.6) as $u_{0}\|\cdot\|^{2} \leq f$, we see through the equality (3.5) that $f=f^{\star, r} \leq$ $h\left(u_{0}\right)\|\cdot\|^{2}=u_{1}\|\cdot\|^{2}$, so $u_{0}\|\cdot\|^{2} \leq f \leq u_{1}\|\cdot\|^{2}$. By induction we derive that

$$
u_{2 n}\|x\|^{2} \leq f(x) \leq u_{2 n+1}\|x\|^{2} \quad \text { for all } n \in \mathbb{N}, x \in X
$$

Observe that for all $t \in I$,

$$
(h \circ h)(t)-t=-\frac{g(t)}{2 r^{2}+4 r t+2} .
$$

Thanks to the inclusion $\left.u_{0} \in\right] \beta, \alpha\left[\cap I\right.$, we have $g\left(u_{0}\right) \leq 0$, and hence

$$
0 \leq(h \circ h)\left(u_{0}\right)-u_{0}=u_{2}-u_{0} .
$$

Consequently, the sequence $\left(u_{2 n}\right)_{n \geq 0}$ is nondecreasing, and then (see the definition of $\left.\left(u_{n}\right)_{n \geq 0}\right)$ we have the fact that $\left(u_{2 n+1}\right)_{n \geq 0}$ is nonincreasing. Coming back to (3.7), we get that $\left(u_{2 n}\right)_{n \geq 0}$ (resp. $\left.\left(u_{2 n+1}\right)_{n \geq 0}\right)$ is bounded from above (resp. below). We deduce that $u_{2 n} \rightarrow \alpha$ and $u_{2 n+1} \rightarrow \alpha$ as $n \rightarrow+\infty$. It remains to let $n \rightarrow \infty$ in (3.7) to complete the proof of the proposition.

Remark 3.2 Let $S$ be an $r$-prox-regular subset of $X$ with $r>0$. Let also $C$ be any nonempty closed convex subset of $X$ with $C \subset U_{s}(S)$ for some real $0<s<r$. It is known (see, e.g., $[5,34])$ that the distance function $d_{S}(\cdot)$ is $(r-s)^{-1}$-semiconvex on $C$, or equivalently $d_{S}+\psi_{C}$ is semiconvex on $X$. This along with Proposition 3.4(j) allows us to write

$$
\left(d_{S}+\psi_{C}\right)(x)=\sup _{x^{\star} \in X}\left(q_{x^{\star}, r-s}(x)-\left(d_{S}+\psi_{C}\right)^{\star, r-s}\left(x^{\star}\right)\right) \quad \text { for all } x \in X .
$$


Consequently, for any $x \in C$ we have

$$
\begin{aligned}
d_{S}(x) & =\sup _{x^{\star} \in X}\left(q_{x^{\star}, r-s}(x)-\sup _{y \in X}\left(\left\langle x^{\star}, y\right\rangle-\frac{\|y\|^{2}}{2(r-s)}-\left(d_{S}+\psi_{C}\right)(y)\right)\right) \\
& =\sup _{x^{\star} \in X}\left(q_{x^{\star}, r-s}(x)-\sup _{y \in C}\left(\left\langle x^{\star}, y\right\rangle-\frac{\|y\|^{2}}{2(r-s)}-d_{S}(y)\right)\right) \\
& =\sup _{x^{\star} \in X} \inf _{y \in C}\left(q_{x^{\star}, r-s}(x)-q_{x^{\star}, r-s}(y)+d_{S}(y)\right) .
\end{aligned}
$$

Now, assume for a moment that the nonempty closed set $S$ is convex and take any $x \in X$. The latter equalities with $C:=X$ give

$$
d_{S}(x)=\sup _{x^{\star} \in X} \inf _{y \in X}\left(\left\langle x^{\star}, x-y\right\rangle+d_{S}(y)\right) .
$$

Writting

$$
\begin{aligned}
\inf _{y \in X}\left(\left\langle x^{\star}, x-y\right\rangle+d_{S}(y)\right) & =\inf _{u \in S}\left(\left\langle x^{\star}, x-u\right\rangle-\sup _{y \in X}\left[\left\langle x^{*}, y-u\right\rangle-\|y-u\|\right]\right) \\
& =\inf _{u \in S}\left(\left\langle x^{*}, x-u\right\rangle-\psi_{\mathbb{B}_{X}}\left(x^{*}\right)\right),
\end{aligned}
$$

we then obtain the known formula (see, e.g., [18, Remarks 7.2,p.126])

$$
d_{S}(x)=\sup _{x^{\star} \in \mathbb{B}_{X}} \inf _{y \in S}\left\langle x^{\star}, x-y\right\rangle=\max _{x^{\star} \in \mathbb{B}_{X}} \inf _{y \in S}\left\langle x^{\star}, x-y\right\rangle,
$$

where the latter equality is due to the weak upper semicontinuity of $x^{\star} \mapsto\left\langle x^{\star}, x-y\right\rangle$. We mention here that an extension of the latter inf-sup formula to the context of prox-regular sets is established in our paper [3].

\section{Prox-regularity and functional separation}

It has been well-recognized that the geometric Hahn-Banach theorems are among of the most important and powerful principles of functional analysis (see, e.g., [19, 18, 27, 33] and the references therein). Roughly speaking, the geometric Hahn-Banach theorem for closed convex sets asserts that a compact convex set $A$ and a closed convex set $B$ of $X$ (or more generally of a locally convex space) with $A \cap B=\emptyset$ can be separated by a hyperplane/half-space. The case where $A$ is reduced to a singleton (so $A$ is an exterior point of $B$ ) is of a great interest and can be stated as follows (see also [18, Theorem 6.23]).

Theorem 4.1 Let $S$ be a nonempty closed convex set of $X, x \in X \backslash S$. Then, one has with $x^{\star}:=d_{S}(x)^{-1}\left(x-\operatorname{proj}_{S}(x)\right) \in \mathbb{S}$ the following separation property for some $\alpha \in \mathbb{R}$,

$$
S \subset\left\{\left\langle x^{\star}, \cdot\right\rangle\langle\alpha\} \subset \mathcal{H}_{\leq}\left(x^{\star}, \alpha\right):=\left\{\left\langle x^{\star}, \cdot\right\rangle \leq \alpha\right\} .\right.
$$

and

$$
\left\langle x^{\star}, x\right\rangle>\alpha \geq \sup _{y \in S}\left\langle x^{\star}, y\right\rangle=: \sigma\left(x^{\star}, S\right) .
$$

Applying the latter theorem with $S:=B-A$ for two subsets $A, B \subset X$ and $x:=0$ leads to the following general convex separation result:

Theorem 4.2 Let $A$ and $B$ be two nonempty disjoint closed and convex sets in $X$ with $A+B$ closed (which holds if $B$ is weakly compact). Then, there exist $x^{\star} \in X$ and a real $\alpha>0$ such that

$$
\sup _{a \in A}\left\langle x^{\star}, a\right\rangle+\alpha \leq \inf _{b \in B}\left\langle x^{\star}, b\right\rangle,
$$

in particular

$$
\sigma\left(x^{\star}, A\right) \leq-\sigma\left(x^{\star},-B\right)=-\sigma\left(-x^{\star}, B\right) .
$$


It is worth noticing that, to argue the two above results, Zorn lemma is not needed because of the Hilbertian structure of $X$.

Our aim in this section is to extend such separation results to the context of $r$-proxregular sets. As expected, the (classical) support function $\sigma_{S}(\cdot)=\varphi_{S, \infty}(\cdot)$ (resp. the linear form $\left.q_{x^{\star}, \infty}(\cdot)=\left\langle x^{\star}, \cdot\right\rangle\right)$ will be replaced by $\varphi_{S, r}(\cdot)$ (resp. the quadratic function $\left.q_{x^{\star}, r}(\cdot)\right)$. Let us mention that going beyond convexity in separation theory is a very challenging issue which has been (apparently) developed in a very few number of works. General nonconvex separation lies at the heart of the construction of Mordukhovich's generalized differentiation with the so-called extremal variational principle (see, e.g., [27, Chapter 2] and the references therein). To the best of our knowledge, the first separation result for prox-regular sets is due to J.P. Vial. In his paper, Vial shows ([37, Theorem 5.1]) in the finite dimensional setting that a weakly convex set of constant $r$ (a concept equivalent to the $r$-prox-regularity in Hilbert spaces) and an $R$-strongly convex set can always be separated by a ball whenever a certain condition holds between the radii $r, R$. Vial also provides an estimate for the radius of the involved ball, depending on $r$ and $R$. Such a result has been successfuly extended to the Hilbert framework by G.E. Ivanov [23] (see also the survey [22]) and by M.V. Balashov and G.E. Ivanov ([6]) with separation result in the context of uniformly convex and smooth Banach spaces. In this section and the next one, we provide other additional separation properties.

Let us start by extending Theorem 4.1 to the context of prox-regular sets. The following easy result will be needed.

Lemma 4.1 Let $S$ be a nonempty closed subset of $X, x \in X \backslash S$. Assume that $p \in$ $\operatorname{Proj}_{S}(x) \neq \emptyset$ and let $\rho$ be an extended real such that $\rho>d_{S}(x)$. The following hold with $x^{\star}:=d_{S}(x)^{-1}(p-x)$.

(a) One has the estimate

$$
\left\langle x^{\star}, x\right\rangle+\frac{1}{\rho} d_{S}^{2}(x)<\left\langle x^{\star}, x\right\rangle+d_{S}(x)=\left\langle x^{\star}, p\right\rangle .
$$

(b) If in addition the set $S$ is $r$-prox-regular for some $r \in] 0,+\infty]$, then for all $y \in S$,

$$
\left\langle x^{\star}, x\right\rangle+\frac{1}{\rho} d_{S}^{2}(x)<\left\langle x^{\star}, p\right\rangle \leq\left\langle x^{\star}, y\right\rangle+\frac{1}{2 r}\|p-y\|^{2} .
$$

Proof. The assertion $(a)$ is a direct consequence of the inequality $\rho^{-1} d_{S}(x)<1$. Let us show $(b)$. Assume that $S$ is $r$-prox-regular for some extended real $r>0$ and fix any $y \in S$. Noting that $-x^{\star} \in N^{P}(S ; p)$ (see $(2.2)$ ), we can apply Theorem 2.1 to get

$$
\left\langle-x^{\star}, y-p\right\rangle \leq \frac{\left\|x^{\star}\right\|}{2 r}\|y-p\|^{2},
$$

or equivalently

$$
\left\langle x^{\star}, p\right\rangle \leq\left\langle x^{\star}, y\right\rangle+\frac{1}{2 r}\|y-p\|^{2} .
$$

Combining (4.1) and (4.2), we arrive to

$$
\left\langle x^{\star}, x\right\rangle+\frac{d_{S}^{2}(x)}{\rho}<\left\langle x^{\star}, p\right\rangle \leq\left\langle x^{\star}, y\right\rangle+\frac{1}{2 r}\|p-y\|^{2} .
$$

The proof is complete.

We derive from the particular case when $S$ is $r$-prox-regular and $r \geq \rho$ the following separation type result.

Proposition 4.1 Let $S$ be an r-prox-regular subset of $X$ with $r \in] 0,+\infty]$. Let $x \in$ $\operatorname{Tube}_{r}(S)$ and $x^{\star}:=d_{S}(x)^{-1}\left(\operatorname{proj}_{S}(x)-x\right)$, and let $\rho>d_{S}(x)$. Then, for all $y \in S$

$$
\left\langle x^{\star}, x\right\rangle+\frac{d_{S}^{2}(x)}{\rho}<\left\langle x^{\star}, \operatorname{proj}_{S}(x)\right\rangle \leq\left\langle x^{\star}, y\right\rangle+\frac{1}{2 r}\left\|\operatorname{proj}_{S}(x)-y\right\|^{2},
$$


so there exists $\alpha \in \mathbb{R}$ such that for all $y \in S$,

$$
\left\langle x^{\star}, x\right\rangle+\frac{d_{S}^{2}(x)}{\rho}<\alpha<\left\langle x^{\star}, y\right\rangle+\frac{1}{2 r}\left\|\operatorname{proj}_{S}(x)-y\right\|^{2} .
$$

Remark 4.1 In addition to the above result, diverse intrinsic metric characterizations of prox-regular sets can be found in $[5,13,30,31,34]$.

In Theorem 4.1, the separation property holds thanks to the vector $x^{\star}=d_{S}(x)^{-1}(x-$ $\left.\operatorname{proj}_{S}(x)\right) \in X \backslash\{0\}$. In the context of $r$-prox-regular sets, the vector $x^{\star}$ will be replaced by $\Gamma_{S, r}(x):=\left(\frac{1}{r}-\frac{1}{d_{S}(x)}\right) \operatorname{proj}_{S}(x)+\frac{1}{d_{S}(x)} x$ (see (3.2)). The next lemma shows in particular that $\Gamma_{S, r}(x) \in X \backslash \frac{1}{r} S$. For $x, y \in X$, we denote as usual $] x, y[:=\{(1-t) x+t y: t \in] 0,1[\}$.

Lemma 4.2 Let $S$ be an $r$-prox-regular subset of $X$ with $r \in] 0,+\infty\left[\right.$ and let $x \in \operatorname{Tube}_{r}(S)$. The following hold with $\left.t:=\frac{d_{S}(x)}{r} \in\right] 0,1[$.

(a) One has

$$
x=\operatorname{tr} \Gamma_{S, r}(x)+(1-t) \operatorname{proj}_{S}(x) \text { and }\left\|\Gamma_{S, r}(x)-\frac{x}{r}\right\|=1-t,
$$

in particular $x \in] \operatorname{proj}_{S}(x), r \Gamma_{S, r}(x)[$.

(b) One has the inclusions

$$
r \Gamma_{S, r}(x)=\left(1-t^{-1}\right) \operatorname{proj}_{S}(x)+t^{-1} x \in D_{r}(S) \quad \text { and } \operatorname{proj}_{S}(x) \in \operatorname{Proj}_{S}\left(r \Gamma_{S, r}(x)\right) .
$$

(c) One has

$$
\left.\operatorname{Tube}_{r}(S)=\bigcup_{u \in \operatorname{Tube}_{r}(S)}\right]_{\operatorname{proj}_{S}(u), r \Gamma_{S, r}(u)[.}
$$

(d) For all $y \in] \operatorname{proj}_{S}(x), r \Gamma_{S, r}(x)[$, one has

$$
\Gamma_{S, r}(x)=\Gamma_{S, r}(y) .
$$

(e) For every $s \in] 0, r[$, one has

$$
\Lambda_{r}(S):=r \Gamma_{S, r}\left(\operatorname{Tube}_{r}(S)\right)=\left\{\left(1-\frac{r}{s}\right) \operatorname{proj}_{S}(y)+\frac{r}{s} y: y \in D_{s}(S)\right\} .
$$

(f) For every $s \in] 0, r[$, one has

$$
\left.\operatorname{Tube}_{r}(S)=\bigcup_{u \in D_{s}(S)}\right] \operatorname{proj}_{S}(u), r \Gamma_{S, r}(u)[.
$$

Proof. (a) It can be checked in a straightforward way.

(b) Observe from Remark 2.1 that with $p:=\operatorname{proj}_{S}(x)$ and $d:=d_{S}(x)$

$$
p \in \operatorname{Proj}_{S}\left(p+r \frac{x-p}{d}\right)=\operatorname{Proj}_{S}\left(r \Gamma_{S, r}(x)\right) .
$$

Consequently, we have $d_{S}\left(r \Gamma_{S, r}(x)\right)=\left\|r \Gamma_{S, r}(x)-p\right\|=t^{-1}\|x-p\|=r$.

(c) According to the assertion (a) above, we know that

$$
\left.\operatorname{Tube}_{r}(S) \subset \bigcup_{u \in \operatorname{Tube}_{r}(S)}\right] \operatorname{proj}_{S}(u), r \Gamma_{S, r}(u)[.
$$

Let us show the converse inclusion. Fix any $u \in \operatorname{Tube}_{r}(S)$ and any $\left.y \in\right] \operatorname{proj}_{S}(u), r \Gamma_{S, r}(u)[$. Set $d:=d_{S}(u)$ and $p:=\operatorname{proj}_{S}(u)$. Let $\left.\lambda \in\right] 0,1\left[\right.$ such that $y=\lambda p+(1-\lambda) r \Gamma_{S, r}(u)$. We obviously have

$$
y=\left(\lambda+(1-\lambda)\left(1-\frac{r}{d}\right)\right) p+(1-\lambda) \frac{r}{d} u=\left(1-(1-\lambda) \frac{r}{d}\right) p+(1-\lambda) \frac{r}{d} u,
$$


hence

$$
\begin{aligned}
d_{S}(y) & =d_{S}\left(\left(1-(1-\lambda) \frac{r}{d}\right) p+(1-\lambda) \frac{r}{d} u\right) \\
& \leq\left\|(1-\lambda) \frac{r}{d}(u-p)\right\|=(1-\lambda) \frac{r}{d} d<r,
\end{aligned}
$$

where the first inequality is due to $p \in S$. By contradiction, suppose that $y \in S$. Then, obviously $\operatorname{proj}_{S}(y)$ is well defined and satisfies $\operatorname{proj}_{S}(y)=y$. On the other hand, the

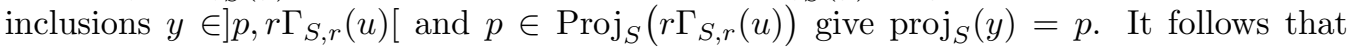
$y=p$ and this is the desired contradiction.

(d) Fix any $y \in] \operatorname{proj}_{S}(x), r \Gamma_{S, r}(x)\left[\right.$. According to (c) above, we have $y \in \operatorname{Tube}_{r}(S)$, in particular $\Gamma_{S, r}(y)$ is well defined. Further, the second inclusion provided by (b) above guarantees that $\operatorname{proj}_{S}(y)=\operatorname{proj}_{S}(x)=: p$. Through elementary computations, we see that

$$
\omega:=\Gamma_{S, r}(x)-\Gamma_{S, r}(y)=\left(\frac{1}{d_{S}(y)}-\frac{1}{d_{S}(x)}\right)(p-x)+\frac{1}{d_{S}(y)}(x-y),
$$

hence

$$
\|\omega\|^{2}=\frac{\left(d_{S}(x)-d_{S}(y)\right)^{2}}{d_{S}^{2}(y)}+\frac{\|x-y\|^{2}}{d_{S}^{2}(y)}+2\left\langle\left(\frac{1}{d_{S}(y)}-\frac{1}{d_{S}(x)}\right)(p-x), \frac{x-y}{d_{S}(y)}\right\rangle .
$$

Now, let us distinguish two cases:

Case 1: $y \in] p, x[$. In such a case, we have

$$
p-x=\frac{d_{S}(x)}{\|x-y\|}(y-x) \quad \text { and } \quad d_{S}(x)=d_{S}(y)+\|x-y\| .
$$

Then, it is not difficult to check that

$$
\left\langle\left(\frac{1}{d_{S}(y)}-\frac{1}{d_{S}(x)}\right)(p-x), \frac{x-y}{d_{S}(y)}\right\rangle=-\frac{\|x-y\|^{2}}{d_{S}^{2}(y)} .
$$

Putting together (4.3), (4.4) and (4.5), we arrive to

$$
\|\omega\|^{2}=\frac{\|x-y\|^{2}}{d_{S}^{2}(y)}+\frac{\|x-y\|^{2}}{d_{S}^{2}(y)}-2 \frac{\|x-y\|^{2}}{d_{S}^{2}(y)}=0
$$

i.e., $\Gamma_{S, r}(y)=\Gamma_{S, r}(x)$.

Case 2: $y \in] x, r \Gamma_{S, r}(x)[$. It is clear that

$$
p-x=\frac{d_{S}(x)}{\|x-y\|}(x-y) \quad \text { and } \quad d_{S}(y)=d_{S}(x)+\|x-y\| .
$$

Proceeding as in Case 1, we obtain $\omega=0$, which is the desired equality.

(e) Fix any $s \in] 0, r\left[\right.$. Observe first that we obviously have with $p_{x}:=\operatorname{proj}_{S}(x)$ and $p_{y}:=\operatorname{proj}_{S}(y)$

$$
\left\{\left(1-\frac{r}{s}\right) p_{y}+\frac{r}{s} y: y \in D_{s}(S)\right\} \subset \Lambda_{r}(S):=\left\{\left(1-\frac{r}{d_{S}(x)}\right) p_{x}+\frac{r}{s} x: x \in \operatorname{Tube}_{s}(S)\right\} .
$$

Let us show the converse inclusion. Let $x \in \operatorname{Tube}_{r}(S)$. Set $v_{x}:=r \Gamma_{S, r}(x)$. Thanks to the continuity of $\theta:[0,1] \rightarrow \mathbb{R}_{+}$defined by

$$
\theta(t):=d_{S}\left(p_{x}+t\left(v_{x}-p_{x}\right)\right) \text { for all } t \in[0,1],
$$

we can choose some $t_{0} \in[0,1]$ such that

$$
y_{0}:=p_{x}+t_{0}\left(v_{x}-p_{x}\right) \in D_{s}(S)
$$


It remains to apply $(d)$ above to get $v_{x}=r \Gamma_{S, r}\left(y_{0}\right)$.

$(f)$ In view of $(c)$, there is only one inclusion to justify. Fix any $\bar{x} \in \operatorname{Tube}_{r}(S)$. By $(a)$ above, we know that $\bar{x} \in] \operatorname{proj}_{S}(\bar{x}), r \Gamma_{S, r}(\bar{x})[$. Defining the function $\theta:[0,1] \rightarrow \mathbb{R}$ by

$$
\theta(t):=d_{S}\left(\operatorname{proj}_{S}(\bar{x})+t\left(r \Gamma_{S, r}(\bar{x})-\operatorname{proj}_{S}(\bar{x})\right)\right) \text { for all } t \in[0,1]
$$

and noticing by (b) above that $\theta(1)=r$, the intermediate value theorem gives some

$$
\left.\bar{y} \in D_{s}(S) \cap\right] \operatorname{proj}_{S}(\bar{x}), r \Gamma_{S, r}(\bar{x})[.
$$

We derive from $(d)$ that $\Gamma_{S, r}(\bar{y})=\Gamma_{S, r}(\bar{x})$. On the other hand, we have $\operatorname{proj}_{S}(\bar{x})=$ $\operatorname{proj}_{S}(\bar{y})$ because $\operatorname{Proj}_{S}\left(r \Gamma_{S, r}(\bar{x})\right) \ni \operatorname{proj}_{S}(\bar{x})$. The inclusion $\left.\bar{x} \in\right] \operatorname{proj}_{S}(\bar{x}), r \Gamma_{S, r}(\bar{x})[$ in (a) can then be rewritten as

$$
\bar{x} \in] \operatorname{proj}_{S}(\bar{y}), r \Gamma_{S, r}(\bar{y})[.
$$

The proof is complete.

With the above results at hands, we are ready to establish a separation theorem by means of $\Gamma_{S, r}(\cdot)$. In order to state the theorem, let us denote for any $x^{\star} \in X, \rho, \alpha \in \mathbb{R}$

$$
Q_{x^{\star}, \rho, \alpha}:=\left\{q_{x^{\star}, \rho} \leq \alpha\right\}:=\left\{x \in X:\left\langle x^{\star}, x\right\rangle-\frac{\|x\|^{2}}{2 \rho} \leq \alpha\right\} .
$$

Theorem 4.3 Let $S$ be an $r$-prox-regular subset of $X$ with $r \in] 0,+\infty], x \in \operatorname{Tube}_{r}(S)$. Then, one has with $x^{\star}:=\Gamma_{S, r}(x)=\left(\frac{1}{r}-\frac{1}{d_{S}(x)}\right) \operatorname{proj}_{S}(x)+\frac{1}{d_{S}(x)} x$ the following separation property for some $\alpha \in \mathbb{R}$

$$
S \subset\left\{\left\langle x^{\star}, \cdot\right\rangle-\frac{\|\cdot\|^{2}}{2 r}<\alpha\right\} \subset Q_{x^{\star}, r, \alpha}
$$

and

$$
q_{x^{\star}, r}(x):=\left\langle x^{\star}, x\right\rangle-\frac{\|x\|^{2}}{2 r}>\alpha \geq \sup q_{x^{\star}, r}(S)=: \varphi_{S, r}\left(x^{\star}\right) .
$$

Proof. Set $p:=\operatorname{proj}_{S}(x), d:=d_{S}(x)$ and $u^{\star}:=d^{-1}(p-x)$. Applying Proposition 4.1 with $\rho:=2 r>d$ gives some $\beta \in \mathbb{R}$ such that for every $y \in S$,

$$
\left\langle u^{\star}, x\right\rangle+\frac{d^{2}}{2 r}<\beta<\left\langle u^{\star}, y\right\rangle+\frac{1}{2 r}\|p-y\|^{2} .
$$

Now, observe that for all $y \in X$,

$$
\left\langle u^{\star}, y\right\rangle+\frac{1}{2 r}\|y-p\|^{2}=\left\langle u^{\star}-\frac{1}{r} p, y\right\rangle+\frac{1}{2 r}\left(\|y\|^{2}+\|p\|^{2}\right) .
$$

Putting the second inequality of (4.8) and the equality (4.9) together gives for all $y \in S$,

$$
\left\langle\frac{1}{r} p-u^{\star}, y\right\rangle-\frac{\|y\|^{2}}{2 r}<\frac{\|p\|^{2}}{2 r}-\beta .
$$

On the other hand, the first inequality of (4.8) and the equality (4.9) yield

$$
\left\langle\frac{1}{r} p-u^{\star}, x\right\rangle-\frac{\|x\|^{2}}{2 r}>\frac{\|p\|^{2}}{2 r}-\beta .
$$

Then, $x^{\star}:=\frac{1}{r} p-u^{\star}=\Gamma_{S, r}(x)$ and $\alpha:=\frac{\|p\|^{2}}{2 r}-\beta$ fulfill the inclusion in (4.6) along with the estimates (4.7). The proof is complete. 
Remark 4.2 It should be noted that we have a similar separation property as (4.6) with reversed inequalities. Indeed, consider any $r$-prox-regular subset $S$ of $X$ with $r \in] 0,+\infty]$ and $x \in U_{r}(S) \backslash S$. It is straightforward to check that $-S$ is also $r$-prox-regular and that the inclusion $-x \in U_{r}(-S) \backslash-S$ holds. Hence, according to Theorem 4.3, we can write for some $u^{\star} \in X$ and some real $\beta$

$$
-S \subset\left\{\left\langle u^{\star}, \cdot\right\rangle-\frac{\|\cdot\|^{2}}{2 r}<\beta\right\} \quad \text { and } \quad\left\langle u^{\star},-x\right\rangle-\frac{\|x\|^{2}}{2 r}>\beta .
$$

Setting $x^{\star}:=-u^{\star}$ and $\alpha:=-\beta$, we obtain

$$
S \subset\left\{\left\langle x^{\star}, \cdot\right\rangle+\frac{\|\cdot\|^{2}}{2 r}>\alpha\right\} \quad \text { and } \quad\left\langle x^{\star}, x\right\rangle+\frac{\|x\|^{2}}{2 r}<\alpha .
$$

As a direct application, we can extend the classical fact that a lower semicontinuous convex function has continuous affine minorants.

Theorem 4.4 Let $f: X \rightarrow \mathbb{R} \cup\{+\infty\}$ be a function with an $r$-prox-regular epigraph for some $r \in] 0,+\infty\left[\right.$. Then, there exist $x^{\star} \in X, \alpha, \theta \in \mathbb{R}$ with $\theta<\sup _{X} \frac{1}{r} f$ such that

$$
\left\langle x^{\star}, x\right\rangle-\frac{\|x\|^{2}}{2 r}+\alpha \leq f(x)\left(\frac{f(x)}{2 r}-\theta\right) \quad \text { for all } x \in X .
$$

Proof. We endow $X \times \mathbb{R}$ with the canonical inner product and we still denote the associated norm by $\|\cdot\|$. If $f \equiv+\infty$ there is nothing to establish, so we may assume that $f \not \equiv+\infty$. Fix any $u \in X$ with $f(u)<+\infty$ and choose any real $0<\varepsilon<r$. Note that $(u, f(u)) \in$ epi $f,(u, f(u)-\varepsilon) \notin$ epi $f$ and

$$
d_{\text {epi } f}(u, f(u)-\varepsilon) \leq \varepsilon<r .
$$

Consequently, we have Tube $_{r}($ epi $f) \cap(\operatorname{dom} f \times \mathbb{R}) \neq \emptyset$. Fix any $(\bar{x}, c) \in$ Tube $_{r}($ epi $f)$ with $f(\bar{x})<+\infty$. According to Theorem 4.3, there are $\left(u^{\star}, \theta\right) \in X \times \mathbb{R}$ and $\alpha \in \mathbb{R}$ such that

$$
\text { epi } f \subset\{\varphi \leq \alpha\} \quad \text { and } \quad(\bar{x}, c) \in\{\varphi>\alpha\}
$$

with $\varphi: X \times \mathbb{R} \rightarrow \mathbb{R}$ defined by

$$
\varphi(x, t):=\left\langle u^{\star}, x\right\rangle+\theta t-\frac{1}{2 r}\|(x, t)\|^{2} \quad \text { for all }(x, t) \in X \times \mathbb{R} .
$$

This says that

$$
\left\langle u^{\star}, x\right\rangle+\theta t-\frac{1}{2 r}\|(x, t)\|^{2} \leq \alpha \quad \text { for all }(x, t) \in \text { epi } f
$$

and

$$
\left\langle u^{\star}, \bar{x}\right\rangle+\theta c-\frac{1}{2 r}\|(\bar{x}, c)\|^{2}>\alpha .
$$

Combining (4.10) and the inclusion $(\bar{x}, f(\bar{x})) \in$ epi $f$, we obtain

$$
\left\langle u^{\star}, \bar{x}\right\rangle+\theta f(\bar{x})-\frac{1}{2 r}\|(\bar{x}, f(\bar{x}))\|^{2} \leq \alpha .
$$

From this and (4.11), we get

$$
\begin{aligned}
\left\langle u^{\star}, \bar{x}\right\rangle+\theta f(\bar{x}) & \leq \frac{1}{2 r}\|(\bar{x}, f(\bar{x}))\|^{2}+\alpha \\
& <\frac{1}{2 r}\|(\bar{x}, f(\bar{x}))\|^{2}+\left\langle u^{\star}, \bar{x}\right\rangle+\theta c-\frac{1}{2 r}\|(\bar{x}, c)\|^{2},
\end{aligned}
$$


hence

$$
\theta f(\bar{x})<\theta c+\frac{1}{2 r}\left(f(\bar{x})^{2}-c^{2}\right) .
$$

On the other hand, using the inequality $f(\bar{x})>c$, we arrive to

$$
\theta<\frac{1}{2 r}(f(\bar{x})+c)<\frac{1}{r} f(\bar{x}) \leq \sup _{X} \frac{1}{r} f .
$$

Finally, taking any $x \in X$ with $f(x) \neq+\infty$ (otherwise, the desired inequality is obvious), it remains to apply (4.10) with the inclusion $(x, f(x)) \in$ epi $f$ to obtain

$$
\left\langle u^{\star}, x\right\rangle-\frac{1}{2 r}\|x\|^{2} \leq \alpha+f(x)\left(\frac{1}{2 r} f(x)-\theta\right) .
$$

The proof is complete.

Remark 4.3 Any $r^{-1}$-semiconvex continuous function $f: X \rightarrow \mathbb{R}$ has its epigraph uniformly prox-regular with constant $r$. Indeed, since $g:=f+\frac{1}{2 r}\|\cdot\|^{2}$ is a continuous convex function, it is locally Lipschitz on $\operatorname{int}(\operatorname{dom} f)=X$ as well as the function $h: X \rightarrow \mathbb{R}$ defined by

$$
h(x, t):=g(x)-\frac{\|x\|^{2}}{2 r}-t \quad \text { for all }(x, t) \in X \times \mathbb{R} .
$$

From the definition of $h$ it can be checked in a straightforward way that

$$
\{h \leq 0\}=\text { epi } f .
$$

On the other hand, we see that

$$
\partial_{C} h(x, t)=\left\{\left(x^{\star}-\frac{x}{r},-1\right): x^{\star} \in \partial_{C} g(x)\right\} \quad \text { for all } x \in X .
$$

Since $g$ is convex, its Clarke subdifferential $\partial_{C} g$ is monotone, hence $\partial_{C} h$ is $\frac{1}{r}$-hypomonotone. It remains to see that for every $\left(x^{\star}, x\right) \in X^{2}$,

$$
\left\langle\left(x^{\star}-\frac{x}{r},-1\right),(0,1)\right\rangle=-1
$$

to apply [1, Theorem 4.1] and get the $r$-prox-regularity of $\{h \leq 0\}$ as desired.

Theorem 4.3 makes clear the important role played by sublevel sets $\left(Q_{x^{\star}, r, \alpha}\right)_{\alpha}$ of the function $q_{x^{\star}, r}$. Such sets are in general nothing but complement of suitable open balls. Indeed, fix any $x^{\star} \in X, \alpha \in \mathbb{R}$ and $\left.r \in\right] 0,+\infty[$. Let $\bar{x} \in X$. Thanks to the elementary equality

$$
\left\|\bar{x}-r x^{\star}\right\|^{2}=r^{2}\left\|x^{\star}\right\|^{2}-2 r\left\langle x^{\star}, \bar{x}\right\rangle+\|\bar{x}\|^{2}
$$

it is easy to observe that

$$
\bar{x} \in Q_{x^{\star}, r, \alpha} \Leftrightarrow\left\|\bar{x}-r x^{\star}\right\|^{2} \geq r^{2}\left\|x^{\star}\right\|^{2}-2 r \alpha .
$$

Hence, we get the following equivalence

$$
\bar{x} \in Q_{x^{\star}, r, \alpha} \Longleftrightarrow \begin{cases}\bar{x} \notin B\left(r x^{\star}, \sqrt{\left.r^{2}\left\|x^{\star}\right\|^{2}-2 r \alpha\right)}\right. & \text { if } r^{2}\left\|x^{\star}\right\|^{2}-2 r \alpha>0 \\ \bar{x} \in X & \text { otherwise. }\end{cases}
$$

If $\alpha=\varphi_{S, r}\left(x^{\star}\right)$ for some closed subset $S$ of $X$, the above equivalence along with the following equalities

$$
\begin{aligned}
r^{2}\left\|x^{\star}\right\|^{2}-2 r \alpha & =r^{2}\left\|x^{\star}\right\|^{2}-2 r \sup _{x \in S}\left(\left\langle x^{\star}, x\right\rangle-\frac{\|x\|^{2}}{2 r}\right) \\
& =-\sup _{x \in S}\left(-r^{2}\left\|x^{\star}\right\|^{2}+2 r\left\langle x^{\star}, x\right\rangle-\frac{\|x\|^{2}}{2 r}\right) \\
& =\inf _{x \in S}\left\|r x^{\star}-x\right\|^{2}=d_{S}^{2}\left(r x^{\star}\right) \geq 0
\end{aligned}
$$


ensure that

$$
Q_{x^{\star}, r, \alpha}= \begin{cases}X \backslash B\left(r x^{\star}, d_{S}\left(r x^{\star}\right)\right) & \text { if } r x^{\star} \notin S, \\ X & \text { otherwise. }\end{cases}
$$

We state the above descriptions of $Q_{x^{\star}, r, \alpha}$ in the following proposition.

Proposition 4.2 Let $x^{\star} \in X, \alpha \in \mathbb{R}$ and $\left.r \in\right] 0,+\infty[$. One has

$$
Q_{x^{\star}, r, \alpha}= \begin{cases}X \backslash B\left(r x^{\star}, \sqrt{r^{2}\left\|x^{\star}\right\|^{2}-2 r \alpha}\right) & \text { if } r^{2}\left\|x^{\star}\right\|^{2}-2 r \alpha>0 \\ X & \text { otherwise. }\end{cases}
$$

In particular, if $\varphi_{S, r}\left(x^{\star}\right)=\alpha \in \mathbb{R}$ for some closed subset $S$ of $X$, one has

$$
r^{2}\left\|x^{\star}\right\|^{2}-2 r \alpha=d_{S}^{2}\left(r x^{\star}\right)
$$

and

$$
Q_{x^{\star}, r, \alpha}= \begin{cases}X \backslash B\left(r x^{\star}, d_{S}\left(r x^{\star}\right)\right) & \text { if } r x^{\star} \notin S, \\ X & \text { otherwise. }\end{cases}
$$

Remark 4.4 Let $S$ be an $r$-prox-regular subset of $X$ with $r \in] 0,+\infty\left[\right.$. Fix $\bar{x} \in \operatorname{bdry}_{X} S$ and choose any $x^{\star} \in N(S ; \bar{x})$. According to Theorem 2.1, for every $x \in S$, we have

$$
\left\langle x^{\star}, x-\bar{x}\right\rangle \leq \frac{\left\|x^{\star}\right\|}{2 r}\|x-\bar{x}\|^{2},
$$

which can be rewritten as

$$
S \subset\left\{x \in X:\left\langle x^{\star}, x-\bar{x}\right\rangle \leq \frac{\left\|x^{\star}\right\|}{2 r}\|x-\bar{x}\|^{2}\right\} .
$$

If $x^{\star}=0$, the latter inclusion is reduced to the evident one $S \subset X$, so assume that $x^{\star} \neq 0$. Set $u^{\star}:=\frac{x^{\star}}{\left\|x^{\star}\right\|}+\frac{\bar{x}}{r}$ and $\alpha:=\left\langle\frac{x^{\star}}{\left\|x^{\star}\right\|}, \bar{x}\right\rangle+\frac{\|\bar{x}\|^{2}}{2 r}$ and note that for each $x \in S$

$$
\begin{aligned}
\left\langle x^{\star}, x-\bar{x}\right\rangle \leq \frac{\left\|x^{\star}\right\|}{2 r}\|x-\bar{x}\|^{2} & \Leftrightarrow\left\langle x^{\star}, x\right\rangle \leq\left\langle x^{\star}, \bar{x}\right\rangle+\frac{\left\|x^{\star}\right\|}{2 r}\left(\|x\|^{2}-2\langle x, \bar{x}\rangle+\|\bar{x}\|^{2}\right) \\
& \Leftrightarrow\left\langle u^{\star}, x\right\rangle-\frac{\|x\|^{2}}{2 r} \leq \alpha,
\end{aligned}
$$

hence

$$
S \subset Q_{u^{\star}, r, \alpha}
$$

On the other hand, it is easy to check that

$$
\rho:=r^{2}\left\|u^{\star}\right\|^{2}-2 r \alpha=r^{2}>0 .
$$

Then, by virtue of Proposition 4.2

$$
S \subset X \backslash B\left(r \frac{x^{\star}}{\left\|x^{\star}\right\|}+\bar{x}, r\right) .
$$

Hence, the set $X \backslash Q_{u^{\star}, r, \alpha}$ is nothing but an $r$-ball which realizes the proximal normal vector $\frac{x^{\star}}{\left\|x^{\star}\right\|}$ to $S$ at $\bar{x}$.

Given $\left.x^{\star} \in X, r \in\right] 0,+\infty\left[\right.$ and $\alpha \in \mathbb{R}$ such that $\rho=r^{2}\left\|x^{\star}\right\|^{2}-2 r \alpha>0$, from the above description of the closed set $Q_{x^{\star}, r, \alpha}$ in Proposition 4.2 says that

$$
Q_{x^{\star}, r, \alpha}=X \backslash B\left(r x^{\star}, \sqrt{\rho}\right),
$$

hence $Q_{x^{\star}, r, \alpha}$ is $\sqrt{\rho}$-prox-regular,

$$
d\left(x, Q_{x^{\star}, r, \alpha}\right)=\left(\sqrt{\rho}-\left\|x-r x^{\star}\right\|\right)^{+} \quad \text { for all } x \in X
$$




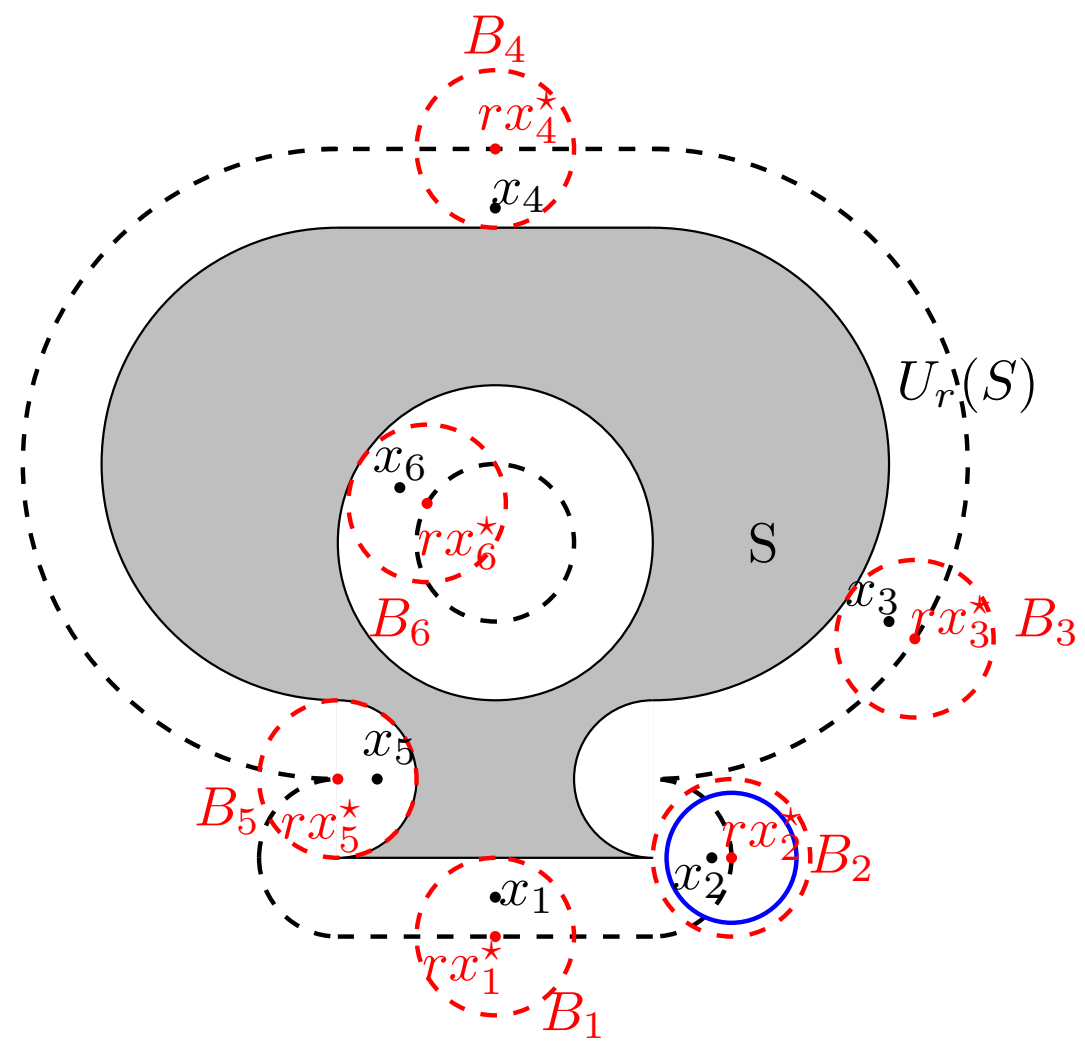

Figure 1: An $r$-prox-regular set with its $r$-open enlargement and some $r$-balls associated to proximal normals. For the point $x_{2}$, the complement of the ball $B_{2}$ illustrates the separation property provided by Theorem 4.3 .

and for every $x \in X \backslash Q_{x^{\star}, r, \alpha}$

$$
\operatorname{Proj}_{Q_{x^{\star}, r, \alpha}}(x)= \begin{cases}S\left(r x^{\star}, \sqrt{\rho}\right) & \text { if } x=r x^{\star}, \\ \left\{\frac{\sqrt{\rho}}{\left\|x-r x^{\star}\right\|}\left(x-r x^{\star}\right)+r x^{\star}\right\} & \text { otherwise. }\end{cases}
$$

It is worth noticing that the above uniformly prox-regular sets $Q_{x^{\star}, r, \alpha}$ are never weakly sequentially closed and never weakly ball-compact whenever the Hilbert space $X$ is infinitedimensional.

Remark 4.5 The $\sqrt{\rho}$-prox-regularity of $Q_{x^{\star}, r, \alpha}$ can also be seen through [1, Theorem 4.1]. Indeed, keep the assumption $\rho>0$ and define the function $f: X \rightarrow \mathbb{R}$ by setting

$$
f(u):=\left\langle x^{\star}, u\right\rangle-\frac{\|u\|^{2}}{2 r}-\alpha \quad \text { for all } u \in X,
$$

so $S=\{f \leq 0\}$. Set $\delta:=\frac{\sqrt{\rho}}{r}>0$ and consider any $x \in$ bdry $Q_{x^{\star}, r, \alpha}$. Choose $w \in \mathbb{B}$ such that

$$
\sup _{v \in \mathbb{B}}\left\langle r x^{\star}-x, v\right\rangle=\left\langle r x^{\star}-x, w\right\rangle=\left\|r x^{\star}-x\right\| .
$$

According to Proposition 4.2, we have $\left\|r x^{\star}-x\right\|=\sqrt{\rho}$, hence with $\bar{v}:=-w \in \mathbb{B}$

$$
\langle\nabla f(x), \bar{v}\rangle=\left\langle x^{\star}-x, \bar{v}\right\rangle=-\frac{\sqrt{\rho}}{r}=-\delta .
$$

A direct application of [1, Theorem 4.1] gives the $r^{\prime}$-prox-regularity of $S=Q_{x^{\star}, r, \alpha}$ with $r^{\prime}:=r \delta=\sqrt{\rho}$. 
Remark 4.6 There is no hope for a kind of Mazur's lemma for prox-regular sets. Besides the non-sequential weak closedness observed above for the uniformly prox-regular sets $Q_{x^{\star}, r, \alpha}$, let us mention that there exist uniformly prox-regular sets which are weakly sequentially closed but not weakly closed. Indeed, with $\left(e_{n}\right)_{n \in \mathbb{N}}$ the natural hilbertian basis of $l_{\mathbb{R}}^{2}(\mathbb{N})$, the set

$$
S:=\left\{\sqrt{n} e_{n}: n \in \mathbb{N}\right\}
$$

is weakly sequentially closed (in particular, strongly closed), not weakly closed [7, Example 3.31] and uniformly prox-regular thanks to the equality

$$
\left\|\sqrt{p} e_{p}-\sqrt{q} e_{q}\right\|=\sqrt{p+q} \geq \sqrt{3} \text { for all } p, q \in \mathbb{N} \text { with } p \neq q .
$$

\section{Ball separation}

This section is devoted to a ball separation for a prox-regular set and a strongly convex set in $X$.

Definition 5.1 Let $C$ be a nonempty closed subset of $X$ and $s \in] 0,+\infty[$. One says that $C$ is s-strongly convex (or strongly convex with constant $s$ ) provided that it is an intersection of closed balls with radius $s>0$, otherwise stated there exists a nonempty set $L$ such that

$$
C=\bigcap_{x \in L} B[x, s]
$$

Let us recall some basic features for such sets.

Theorem 5.1 Let $C$ be a nonempty closed bounded convex subset of $X$ with $C \neq X$ and let $s \in] 0,+\infty[$. The following assertions are equivalent:

(a) The set $C$ is s-strongly convex.

(b) For all $(x, v) \in X^{2}$ with $x \in \operatorname{bdry} C$ and $v \in N(C ; x) \cap \mathbb{S}$, one has

$$
C \subset B[x-s v, s] .
$$

(c) For all $x^{\prime} \in$ bdry $C$ and for all $(x, v) \in X^{2}$ with $x \in \operatorname{bdry} C$ and $v \in N(C ; x) \cap \mathbb{S}$, one has

$$
\left\langle v, x^{\prime}-x\right\rangle \leq-\frac{1}{2 s}\left\|x^{\prime}-x\right\|^{2} .
$$

(d) For all $x, x^{\prime} \in C$ and for all $v \in N(C ; x)$, one has

$$
\left\langle v, x^{\prime}-x\right\rangle \leq-\frac{\|v\|}{2 s}\left\|x^{\prime}-x\right\|^{2} .
$$

Furthermore, any non-singleton strongly closed set has nonempty interior.

The equivalence $(a) \Leftrightarrow(b)$ in the above theorem was proved by J.-P. Vial [36, Theorem 1] and by E.S. Polovinkin and M.V. Balashov [32, Theorem 4.1.2], and $(c)$ is a translation of $(b)$. 1.12.3].

We will need another property which can be found in G.E. Ivanov's book [23, Theorem

Theorem 5.2 Let $S_{1}$ and $S_{2}$ be nonempty sets in $X$. If $S_{1}$ is $r$-prox-regular with $r \in$ ] $0,+\infty]$ and $S_{2}$ is s-strongly convex with $0<s<r$. Then the set $S_{1}+S_{2}$ is closed.

The result in the next proposition is known (see G.E. Ivanov [23, Theorem 1.12. 4]). We provide a simple proof different from that in [23]. Observe first that, given two sets $S_{1}, S_{2}$ in $X$ and $x^{*} \in N^{P}\left(S_{1}+S_{2} ; x\right)$, for any $x_{i} \in S_{i}$ with $x=x_{1}+x_{2}$ it directly results from the definition of proximal normal that $x^{*} \in N^{P}\left(S_{1} ; x_{1}\right) \cap N^{P}\left(S_{2} ; x_{2}\right)$. 
Proposition 5.1 Let $S_{1}$ be an r-prox-regular set in $X$ and $S_{2}$ be a strongly convex set in $X$ with constant $s \in] 0, r\left[\right.$. Then, the set $S_{1}+S_{2}$ is $(r-s)$-prox-regular.

Proof. We know by Theorem 5.2 that $S_{1}+S_{2}$ is closed. Fix any $x \in S_{1}+S_{2}$, so there are $x_{i} \in S_{i}$, with $i=1,2$, such that $x=x_{1}+x_{2}$. Take any $x^{*} \in N^{P}\left(S_{1}+S_{2} ; x\right)$ and any $y \in S_{1}+S_{2}$. Choose $y_{i} \in S_{i}$, with $i=1,2$, such that $y=y_{1}+y_{2}$. We have $x^{*} \in N\left(S_{2} ; x_{2}\right) \cap N^{P}\left(S_{1} ; x_{1}\right)$. Using both (d) in Theorem 5.1 and (b) in Theorem 2.1 we obtain

$$
\left\langle x^{*}, y_{1}+y_{2}-x\right\rangle \leq \frac{\left\|x^{*}\right\|}{2}\left(\frac{1}{r}\left\|y_{1}-x_{1}\right\|^{2}-\frac{1}{s}\left\|y_{2}-x_{2}\right\|^{2}\right) .
$$

Set $a:=y_{1}-x_{1}$ and $b:=y_{2}-x_{2}$ and note that

$$
-2\left\langle\sqrt{\frac{s}{r}} a, \sqrt{\frac{r}{s}} b\right\rangle \leq \frac{s}{r}\|a\|^{2}+\frac{r}{s}\|b\|^{2}, \quad-\frac{s}{r}\|a\|^{2}-\frac{r}{s}\|b\|^{2} \leq 2\langle a, b\rangle,
$$

which entails that

$$
\left(1-\frac{s}{r}\right)\|a\|^{2}+\left(1-\frac{r}{s}\right)\|b\|^{2} \leq\|a\|^{2}+\|b\|^{2}+2\langle a, b\rangle=\|a+b\|^{2} .
$$

The latter inequality means that

$$
\frac{1}{r}\|a\|^{2}-\frac{1}{s}\|b\|^{2} \leq \frac{1}{r-s}\|a+b\|^{2} .
$$

This combined with (5.1) yields $\left\langle x^{*}, y_{1}+y_{2}-x\right\rangle \leq \frac{\left\|x^{*}\right\|}{2(r-s)}\left\|y_{1}+y_{2}-x\right\|^{2}$, which ensures the $(r-s)$-prox-regularity of $S_{1}+S_{2}$ by (b) in Theorem 2.1 again.

We can now give two ball separation results in the next two theorems between a proxregular set and a strongly convex set. Similar results were also established by J.-P. Vial [37, Theorem 5.1] in finite-dimensional Euclidean spaces and by G.E. Ivanov [23, Theorem 1.18.2] in Hilbert spaces.

Theorem 5.3 Let $S$ be an $r$-prox-regular set of the Hilbert space $X$ with $r>0$ and $C$ be a non-singleton closed set in $X$ which is r-strongly convex with $C \cap S=\{\bar{x}\}$ and $\bar{x} \in \operatorname{bdry} C$. Then one has the ball separation property

$$
C \subset B[\bar{x}-r v, r] \quad \text { and } \quad B(\bar{x}-r v, r) \cap S=\emptyset .
$$

Proof. By Theorem 5.1 we know that $\operatorname{int} C \neq \emptyset$. Let us first show that $(-\bar{x}+\operatorname{int} C) \cap$ $T^{B}(S ; \bar{x})=\emptyset$. Indeed, suppose there is some $h$ in the latter intersection. There are sequences $\left(t_{n}\right)_{n}$ tending to 0 with $0<t_{n}<1$ and $\left(h_{n}\right)_{n}$ converging to $h$ in $X$ such that $\bar{x}+t_{n} h_{n} \in S$ for all $n \in \mathbb{N}$. For $n$ large enough, say $n \geq N$, we have $h_{n} \in-\bar{x}+\operatorname{int} C$, hence (since $0 \in \operatorname{cl}(-\bar{x}+\operatorname{int} C)$ ) we see that $t_{n} h_{n} \in-\bar{x}+\operatorname{int} C$. It ensues that $\bar{x}+t_{N} h_{N} \in$ $S \cap \operatorname{int} C$, which is a contradiction.

By the emptiness of the above intersection and the Hahn-Banach separation property there is some $v \in X$ with $\|v\|=1$ such that

$$
\langle v,-\bar{x}+z\rangle<0 \forall z \in \operatorname{int} C \text { and }\langle v, h\rangle \geq 0 \forall h \in T^{B}(S ; \bar{x})=T^{C}(S ; \bar{x}),
$$

keep in mind that $T^{C}(S ; \bar{x})$ is a closed convex cone and $0 \in \operatorname{cl}(-\bar{x}+C)$. Using the equalities $\left(T^{C}(S ; \bar{x})\right)^{\circ}=N^{C}(S ; \bar{x})=N^{P}(S ; \bar{x})$, it results that $v \in N(C ; \bar{x})$ and $-v \in N^{P}(S ; \bar{x})$, thus by (b) in Theorem 5.1 and by Definition 2.1 combined with (2.4) we have

$$
C \subset B[\bar{x}-r v, r] \quad \text { and } \quad S \cap B(\bar{x}-r v, r)=\emptyset,
$$

which is the desired separation property.

Theorem 5.4 Let $S$ be an $r$-prox-regular set of the Hilbert space $X$ with $r>0$ and $C$ be a closed set in $X$ which is s-strongly convex with $0<s<r$. For $g:=\operatorname{gap}(S, C)$, where $\operatorname{gap}(S, C):=\inf \{\|x-y\|: x \in S, y \in C\}$, assume that $0<g<r-s$. Then there exists $a \in X$ such that

$$
C \subset B[a, s] \text { and } S \cap B(a, s+g)=\emptyset,
$$

and the latter equality entails in particular $S \cap B[a, s]=\emptyset$. 
Proof. By Proposition 5.1 the set $S-C$ is $(r-s)$-prox-regular and $0 \notin S-C$ with $d(0, S-C)=g<r-s$. The point 0 then admits a (unique) nearest point $\bar{z} \in S-C$. Write $\bar{z}=\bar{x}-\bar{y}$ with $\bar{x} \in S$ and $\bar{y} \in C$, so $g=\|\bar{x}-\bar{y}\|$. Note that $\bar{x}$ (resp. $\bar{y}$ ) is a nearest point of $\bar{y}$ (resp. $\bar{x}$ ) in $S$ (resp. $C$ ). Setting $v:=(\bar{y}-\bar{x}) /\|\bar{y}-\bar{x}\|$, we see that $v$ belongs to $N(C ; \bar{y})$ and $N^{P}(S ; \bar{x})$. By (b) in Theorem 5.1 and by Definition 2.1 combined with (2.4) we have

$$
C \subset B[\bar{y}+s v, s] \quad \text { and } \quad S \cap B(\bar{x}+r v, r)=\emptyset .
$$

It remains to prove the inclusion $B(\bar{y}+s v, s+g) \subset B(\bar{x}+r v, r)$. Take any $y \in B(\bar{y}+s v, s+g)$ and note that

$$
\begin{aligned}
\|y-(\bar{x}+r v)\| & =\|(y-(\bar{y}+s v))+(\bar{y}-\bar{x}+s v-r v)\| \\
& <s+g+\left\|(\bar{y}-\bar{x})\left(1-\frac{r-s}{g}\right)\right\|,
\end{aligned}
$$

then $\|y-(\bar{x}+r v)\|<s+g+g\left(\frac{r-s}{g}-1\right)=r$, which confirms that $B(\bar{y}+s v, s+g) \subset$ $B(\bar{x}+r v, r)$. This finishes the proof of the theorem.

Acknowledgement. The second author has received funding from the European Union's Horizon 2020 Research and Innovation Programme under the Marie SklodowskaCurie Grant Agreement No 823731 CONMECH.

\section{References}

[1] S. Adly, F. Nacry, L. Thibault, Preservation of Prox-Regularity of Sets with Applications to Constrained Optimization, SIAM J. Optim. 26 (2016), 448-473.

[2] S. Adly, F. Nacry, L. Thibault, Prox-regularity approach to generalized equations and image projection, ESAIM: COCV, 24 (2018), 677-708.

[3] S. Adly, F. Nacry, L. Thibault, New metric properties for prox-regular sets, submitted.

[4] E. Asplund, C̆ebyšev sets in Hilbert space, Trans. Amer. Math. Soc. 144 (1969), 235240.

[5] M.V. Balashov, Weak convexity of the distance function, J. Convex Anal, 20 (2013), 93-106.

[6] M.V. Balashov, G.E. Ivanov, Weakly convex and proximally smooth sets in Banach spaces, Izv. Math. 73 (2009), 455-499.

[7] H.H Bauschke, P.L. Combettes, Convex Analysis and Monotone Operator Theory in Hilbert Spaces, Springer New-York, 2011.

[8] H. Berens, Best approximation in Hilbert space, in Approximation Theory III (Proc. Conf., Univ. Texas, Austin, Tex., 1980) (E. W. Cheney, ed.), Academic Press, New York (1980), 1-20.

[9] F. Bernard, L. Thibault, N. Zlateva Characterizations of prox-regular sets in uniformly convex Banach spaces, J. Convex Anal. 13 (2006), 525-559.

[10] F. Bernard, L. Thibault, N. Zlateva, Prox-regular sets and epigraphs in uniformly convex Banach spaces: various regularities and other properties, Trans. Amer. Math. Soc. 363 (2011), 2211-2247.

[11] E. Bishop, R.R. Phelps, The support functionals of convex sets, in Convexity, edited by V. Klee, vol. VII of Proceedings of Symposia in Pure Mathematics, pp. 27-35, American Mathematical Society, Providence, Rhode Island, 1963. 
[12] J.M. Borwein, J.D. Vanderwerff, Convex Functions: constructions, characterizations and counterexamples, Encyclopedia of Mathematics and its Applications, 109. Cambridge University Press, Cambridge, 2010.

[13] M. Bounkhel, L. Thibault, Nonconvex sweeping process and prox-regularity in Hilbert space, Journal of Nonlinear and Convex Analysis, 6 (2005), 359-374.

[14] P. Cannarsa, C. Sinestrari, Semiconcave Functions, Hamilton-Jacobi Equations, and Optimal Control, Birkhäuser, Boston (2004).

[15] F.H. Clarke, Optimization and Nonsmooth Analysis, Second Edition, Classics in Applied Mathematics, 5, Society for Industrial and Applied Mathematics (SIAM), Philadelphia, PA, 1990.

[16] F.H. Clarke, R.J. Stern, P.R. Wolenski, Proximal smoothness and the lower-C $C^{2}$ property, J. Convex Anal (1995), 117-144.

[17] G. Colombo, L. Thibault, Prox-regular sets and applications, Handbook of nonconvex analysis and applications 99-182 (2010), Int. Press, Somerville, MA.

[18] F.R. Deutsch, Best Approximations in Inner Product Spaces, CMS Books in Mathematics/Ouvrages de Mathématiques de la SMC, 7, Springer-Verlag, New-York, 2001.

[19] M. Fabian, P. Habala, P. Hajek, V. Montesinos, V. Zizler, Banach Space Theory. The Basis for Linear and Nonlinear Analysis., CMS Books in Mathematics/Ouvrages de Mathmatiques de la SMC. Springer, New York, 2011.

[20] H. Federer, Curvature measures, Trans. Amer. Math. Soc. 93 (1959), 418-491.

[21] W. Fenchel, On conjugate convex functions, Canad. J. Math. 1 (1949), 73-77.

[22] V.V. Goncharov, G. E. Ivanov, Strong and weak convexity of closed sets in a Hilbert space, Operations research, engineering, and cyber security, 259-297, Springer Optim. Appl., 113, Springer, Cham, 2017).

[23] G.E. Ivanov, Weakly Convex Sets and Functions: Theory and Applications, Fizmatlit, Moscow, 2006 (in Russian).

[24] J.-B. Hiriart-Urruty, Ensembles de Tchebychev vs ensembles convexes : l'état de la situation vu via l'analyse convexe non lisse, Ann. Sci. Math. Québec 22 (1998), 47-62.

[25] J.-B. Hiriart-Urruty, La conjecture des points les plus éloignés revisités, Ann. Sci. Math. Québec 29 (2005), 197-214.

[26] A.-M. Legendre, Mémoire sur l'intégration de quelques équations aux différences partielles, Histoire de l'Académie royale des sciences (1787), 309-351.

[27] B.S. Mordukhovich, Variational Analysis and Generalized Differentiation I, Grundlehren Series Vol. 330, Springer, 2006.

[28] J.J. Moreau, Fonctionnelles Convexes, Second edition, Universita di Roma "Tor Vergata", 2003.

[29] F. Nacry, L. Thibault, Regularization of sweeping process: old and new, Pure and Applied Functional Analysis, 4, 59-117 (2019).

[30] F. Nacry, L. Thibault, Distance function from a prox-regular set, available soon on Arxiv.

[31] R.A. Poliquin, R.T. Rockafellar, L. Thibault, Local differentiability of distance functions, Trans. Amer. Math. Soc. 352 (2000), 5231-5249.

[32] E.S. Polovinkin and M.V. Balashov, Elements of Convex and Strongly Convex Analysis, Fizmatlit, Moscow, 2004 (in Russian). 
[33] R.T. Rockafellar, R.J.-B. Wets, Variational Analysis, Grundlehren der Mathematischen Wissenschaften, vol. 317. Springer, New York, 1998.

[34] L. Thibault, Unilateral Variational Analysis in Banach Spaces. To appear soon.

[35] J. Venel, A numerical scheme for a class of sweeping processes, Numer. Math. 118 (2011), 367-400.

[36] J.-P. Vial, Srong convexity of sets and functions, J. Mathematical Economics 19 (1982), 187-205.

[37] J.-P. Vial, Strong and weak convexity of sets and functions, Math. Oper. Res. 8 (1983), 231-259. 